\title{
Marine molluscs from Cape Milazzo (Sicily, Italy): a baseline
}

\author{
Salvatore Giacobbe' \& Giuseppe Notaristefano²
}

'Department of Chemical, Biological, Pharmaceutical and Environmental Sciences, University of Messina, Viale F. Stagno d'Alcontres 31, 98166 Messina, Italy; e-mail: sgiacobbe@unime.it

${ }^{2}$ Via G. D’Annunzio 23, 98057 Milazzo, Messina, Italy; e-mail: gnotaristefano@alice.it

ABSTRACT An original data set of 556 benthic mollusc taxa, as a first account of Cape Milazzo local biodiversity, is provided. Qualitative differences between more or less anthropized areas have been put in evidence, and species distribution according to the main habitat typologies has been detailed. The highest biodiversity was found in the northern sites, corresponding to the Zone A of the established marine protected area. Most mollusc species under the European Economic Community (EEC) and National protection, and other ones listed as threatened, have been recorded, and are mainly associated to priority habitats as phanerogams meadows and vermitid reefs. In the meantime, the settlement of not indigenous species, mainly of tropical origin, and disease affecting threatened organisms under EEC protection, testified the vulnerability of the local ecosystem under the global change threat.

KEY WORDS Mediterranean; molluscs; marine reserves; biodiversity; threatened species.

Received 10.08.2018; accepted 30.08.2018; printed 30.09.2018; published online 05.10.2018

\section{INTRODUCTION}

The naturalistic heritage of Cape Milazzo has long been recognized and in May 2018 it lead to the establishment of a marine protected area. Scientific literature, however, is poor in information concerning the local marine biodiversity, especially on what concerns mollusc fauna, whose contribution is almost unknown. On the contrary, the fossil mollusc assemblages have been widely described (e.g., Ruggeri \& Greco, 1965).

Current knowledge about the just declared marine protected area mainly regards coastal fishes assemblages, whose assessment in some priority habitats, as rocky algal reefs and Posidonia oceanica (L.) Delile meadows (UNEP-MAP$\mathrm{RAC} / \mathrm{SPA}, 2006$ ), has been recently carried out in support of zoning proposal (La Mesa et al., 2017). Benthic fish assemblages are also known from near-shore vermetid reefs (Consoli et al., 2008). A further priority habitat of builder molluscs, Dendropoma cristatum (Biondi, 1859), has been studied in their genetic/biogeographic features (Templado et al., 2016). Benthic fauna from marine caves has been recently investigated by Scotti et al. (2017), which reported 37 taxa belonging to poriferans, cnidarians, annelids, and briozoans, although without mentioning molluses. Overall, 55 species of molluscs have been reported in the past by Bombace $(1969,1970)$ from coralligenous seafloors, while 89 species have been recently reported by D'Alessandro et al. (2016) from a wide area of the Milazzo Gulf outside the proposed protected area. Dated sporadic records (Di Natale, 1982) have concerned some introduced taxa, namely the gastropod Cerithium scabridum Philippi, 1848 and the bivalve Pinctada imbricata radiata (Leach, 1814). 
The aim of this paper is to provide original data on Cape Milazzo mollusc fauna and to constitute a baseline on which future dynamics of marine biodiversity both in protected and not protected areas can be evaluated.

\section{MATERIAL AND METHODS}

\section{Study area}

Cape Milazzo, located in the north-eastern coast of Sicily (Central Mediterranean), extends northward towards the Tyrrhenian Sea for about 6 $\mathrm{km}$ and stretches to a maximum width of $1.5 \mathrm{~km}$ (Fig. 1). The coastline is characterized by high rocky cliffs with cobble pocket beaches, due to high hydrodynamism and wave exposure, especially in the northern and western promontory's sides. Steep rocky bottoms prevail, but also sandgravel inlets and patchy distributed Posidonia oceanica beds are widely diffused. South-eastern coasts are submitted to a lower water circulation, mainly flowing eastward, which generates an area of sediment accumulation (Sitran et al., 2009). Close to the promontory, anthropogenic pressure is high, mainly due to the presence of oil refineries and associated marine traffic. Impacts on marine environment have been investigated by Bergamasco et al. (2014), D'Alessandro et al. (2016), Di Bella et al. (2018), but only marginally they have been involved with the promontory coastal waters.

Meanwhile, issues related to the management of the Marine Protected Area have already been addressed, for example regarding the interference with small-scale fisheries (Battaglia et al., 2017).

The proposed zonation of the reserve includes: a zone $\mathrm{A}$, in which no activity other than those authorized for the purpose of scientific research would be permitted; a zone $\mathrm{B}$, with less restrictive regulation, except in the sub-sector Bs where the limitations would be greater; and a zone $\mathrm{C}$, in which would be forbidden only underwater fishing and some recreational sporting activities such as water skiing or jet skiing.

The zone A, at the extreme north of the Cape, is mainly characterized by relevant coralligenous formations (Bombace 1969, 1970). The almost discontinuous zone $\mathrm{B}$, in the north, includes the seabed surrounding "Punta Mazza", a stretch of the east coast between Cirucco Point and Rugno Point, the Bay of S. Antonio to north-west, and a short stretch of coastline near the "Testa dell'Impiccato", southernmost. The sub-area Bs, to the north-west, includes a jagged stretch of coast with the so called West Shoal, Portella reef, and several small rocks and shoals.

\section{Methods}

A checklist of benthic mollusc species has been compiled according to WoRMS (http:/Www. marinespecies.org/), and updated at 2018-07-30, employing data collected in various contexts and research programs. The whole data set has been organized according to the proposal of reserve zonation, but including also some areas that should be considered of naturalistic value although not considered in the protection plan. Areas impacted by various anthropic activities have also been considered. Overall, five sites have been distinguished, as shown in figure 1:

Site 1. Area facing the industrial plants of Milazzo, the Mediterranean Refinery, and the thermoelectric power plant. Samples have been collected by diving within $10 \mathrm{~m}$ of depth.

Site 2. Industrial port of Milazzo. Samples have been collected by scraping iron pillars, from $3 \mathrm{~m}$ to $7 \mathrm{~m}$ depth, in September 2010. Other data have been collected by washing overall $70 \mathrm{dm} 3$ of mudsandy sediment sampled from June 2015 to June 2016.

Site 3. East coast, from the Marina of "Vaccarella" up to Cala Oliva Point. Samples collected by diving within $10 \mathrm{~m}$ of depth, on sandy bottom with emerging rock slabs and patch-distributed Posidonia oceanica meadows.

Site 4. North-eastern coast, characterized by a short stretch of sandy beach alternating with small rocky cliffs from Riva Smeralda to Cirucco point. Samples have been collected by diving within $10 \mathrm{~m}$ of depth, on sandy bottoms with emerging rock slabs and patch-distributed Posidonia oceanica meadows. Further data have been collected by dredging between $50 \mathrm{~m}$ and $60 \mathrm{~m}$ depth.

Site 5. Northern coast, corresponding to the proposed zone A (integral reserve). Samples have been manually collected from reef pools in Mazza Point (a); from remainders on fishing nets operat- 
ing on mud-detrital floors, 180-200 m depth, 2 NM NE from Mazza Point (b) and similarly 2 NM NW from Gamba di Donna Point (c); by SCUBA divers on hard bottom from "Scoglio della Portella", 25$35 \mathrm{~m}$ depth (d) and from Cala S. Antonino, on patch distributed Posidonia oceanica meadows, 20 m depth.

Each record, moreover, has been contextualized in a simplified habitat typology, as follows:

PA - Photophilic Algae, on rocky cliff or cobbles, eventually trapping sand deposits.

PM - Phanerogam Meadows, mainly Posidonia oceanica on sandy or rocky bottoms, most rarely Cymodocea nodosa (Ucria) Asch. on sandy or sandmuddy bottoms.

RP - Reef Pools.

CD - Coastal Detritic and Biodetritic bottoms, including deep coralligenous formations.

SR - Supratidal Rocky reef.

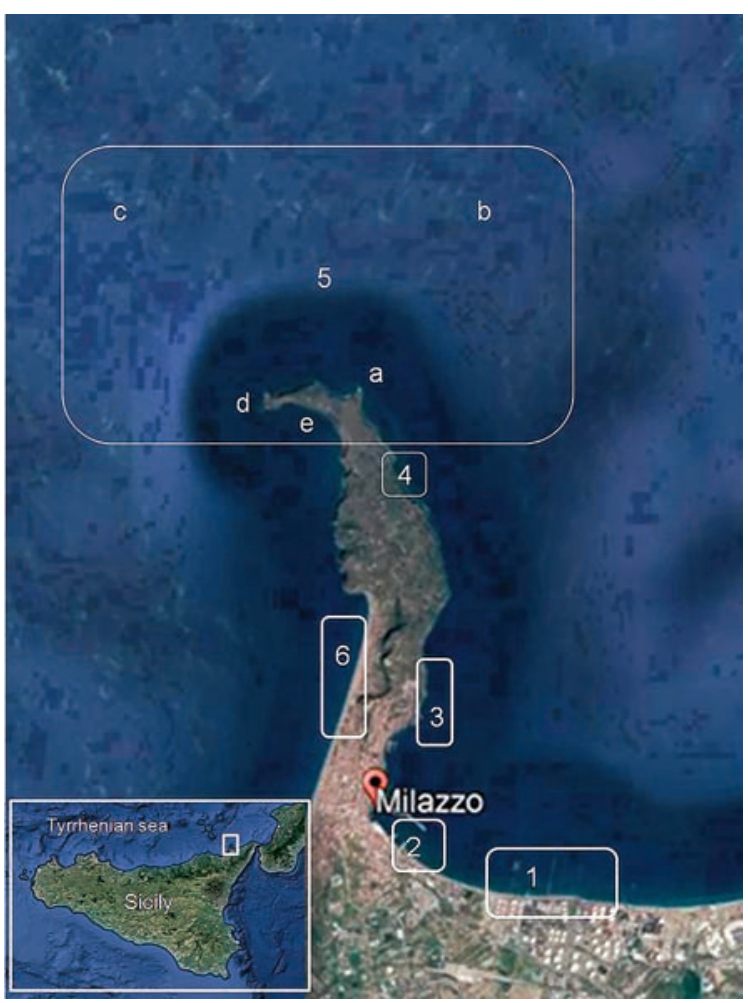

Figure 1. Cape Milazzo: the monitored sites (1-6) and the sub-areas of site 5 (a-e) are indicated.
Collections of stranded pelagic species have been moreover carried out in the five sites, but also in the western coast (site 6), due to particularly favorable wind exposure.

\section{RESULTS AND DISCUSSION}

The monitoring of Cape Milazzo seafloors overall provided 556 taxa, including 9 polyplacophorans, 392 gastropods, 152 bivalves, and 4 scaphopods, whose distribution per site and habitat type is arranged in Table 1. High numbers of species were found in the harbor area (Site 2), as well as in the Riva Smeralda (Site 4), which counted 243 and 217 taxa respectively. Site 3, close to the City of Milazzo, has provided 128 species, whilst just 57 species have been reported from the seafloors facing the industrial pole (Site 1).

The northernmost area, Site 5, was the most biodiverse, counting 155 species found in the sub-area $5 \mathrm{c}, 142$ species in $5 \mathrm{~b}$, and 139 in $5 \mathrm{~d}$. Only 12 species have been recorded in Cala S. Antonino (5e) occasional samplings, while the low diversity (33 species) found in 5a supported with the reef pool (RP) extreme conditions. The Littorinidae Melarhaphe neritoides (Linnaeus, 1758) and Echinolittorina punctata (Gmelin, 1791) were the only mollusc species recorded in the extreme habitat of the supratidal rocky cliff (SR). The phanerogam meadow habitat (PM) showed, as expected, the highest biodiversity (302 species), followed by the photophilc algae (PA) and coastal detritic (CD) environments, which counted almost the same number of species (259 and 267, respectively). CD shared a notable number of species with the coralligenous formations investigated in the past by Bombace $(1969,1970)$, which listed 55 species of gastropods and 35 of bivalves, of which only three species were lacking in the present investigation, i.e., the gastropod Babelomurex cariniferus (Sowerby, 1834), reported as Coralliophila babelis (Requien, 1848), and the bivalves Globivenus effossa (Philippi, 1836) and Coralliophaga lithophagella (Lamarck, 1819).

The most interesting species recorded in the proposed zone A was a fresh empty shell of Haliella tyrrenica Di Geronimo et La Perna, 1999, collected in spring 2011 (site 5c). This species (Fig. 2), initially suspected to be an holocene subfossil taxa since it has never been found alive, has been re- 
cently reported from various localities of the Tuscan Archipelago and, according to Giusti \& Micali (2018), might be actually living. The rather fresh specimen figured by Romani et al. (2016) and the present record are in agreement with such hypothesis.

Among the most widely distributed species, the Mediterranean endemic fan shell, Pinna nobilis Linnaeus, 1758, deserves particular attention, with a large population extending from Punta di Croce di Mare to Punta Cala Oliva, starting from $2 \mathrm{~m}$ depth. In the same stretch of seafloor, sporadic specimens of $P$. rudis Linnaeus, 1758, also occurred. Both fan shells, which play an important role as habitat builders, are protected species under the European Council Directive 92/43/EEC (Eurpean Economic Community, EEC, 1992), in the ANNEX II of Barcelona Convention and by local law in all the Mediterranean countries of the European Union. Their occurrence in the coastal waters of Milazzo is thus remarkable in the framework of establishing the marine protected area. Mass mortality events that recently affected wide areas in the western Mediterranean, including Cape Milazzo (Cabanellas-Reboredo et al., submitted) further suggest the needing of accurate monitoring of their local populations. Other species under EEC and National protection were the date mussel Lithophaga lithophaga (Linnaeus, 1758), widely distributed along the rocky cliffs, the whelks Charonia lampas (Linnaeus, 1758) and Ranella olearia (Linnaeus, 1758), both recorded in the proposed "zone A" (site 5c), and the cowries Naria spurca (Linnaeus, 1758) and Luria lurida (Linnaeus, 1758), both apparently localized in Site 3 (Vaccarella-Puntaloro-Cala Oliva). The vermetid Dendropoma cristatum (Biondi, 1859) is reported in both the eastern and northern coasts (Sites 4, 4, and $5 \mathrm{~d}$ ), where it forms relevant bioconstructions. Species of high interest were also the whelks Monoplex parthenopeus (Salis Marschlins, 1793) (Fig. 3), which is included in the "Red List" of threatened species, and Bursa scrobilator (Linnaeus, 1758), not yet considered as endangered, although infrequent in the Mediterranean and actively sought for collection purposes.

Haliotis stomatiaeformis Reeve, 1846 (Fig. 4) is a poorly known species reported only from continental Sicily up to Malta (Geiger, 2000). According to Gaeta et al. (2003), habitat selection might be the ecological factor allowing its coexistence with the common $H$. tuberculata tuberculata Linnaeus, 1758 .

The record of Mathilda bielerei Smriglio et Mariottini, 2007 (Figs. 5-7), a species not yet dealt with in the scientific literature after its original description in Smriglio et al. (2007), is of relevant interest since it testifies that such species is not strictly tied to the type habitat, the deep-sea coral banks.

The settlement of a small population of the pearl oyster Pinctata imbricata radiata (Leach, 1814) (Fig. 8) is of different interest, as it testifies the progressive consolidation and spreading in the western basin of this species of Lessepsian origin, long time naturalized in the eastern basin up to the Ionian coasts of Sicily, with dated occasional reports from Milazzo (Di Natale, 1982) and sporadic records in the western Mediterranean (Lodola et al., 2013). Long time naturalized Lessepsian is also the mussel Brachidontes pharaonis (P. Fischer, 1870), whose spread in the central Mediterranean has been initially documented from western Sicily (Sarà et al., 2008). The occurrence of the warm Atlantic sea hare Aplysia dactylomela Rang, 1828, spreading in Tyrrhenian sea from Messina Strait since 2011 (Valdés et al., 2013) is a further evidence of ongoing tropicalization processes.

The record, at last, of single shells of Buccinum undatum (Linnaeus, 1758), Nassarius turulosus (Risso, 1826), and Euspira catena (da Costa,1778), not included in the present list, suggested the occurrence of offshore würmian age deposits.

On top of the benthic species, other 14 pelagic molluscs have been recorded in the coastal waters of Cape Milazzo. Most of them were shell remains of pteropoda found in bottom sediments of Site 5 (Tab. 2). More interesting, since specimens have been recorded living, were the violet snails stranded along both the eastern (Sites 2 and 3 ) and western coasts (site 6). Two species have been collected, the common Janthina pallida Thompson, 1840, of which massive stranding have been recorded, e.g., in October 1998, and the much less common J. globosa Swainson, 1822, which is suspected to be in further rarefaction. Stranding of the pseudothecosomata Cymbulia peronii Blainville, 1818 , has been also documented along the western coast (Site 6). 

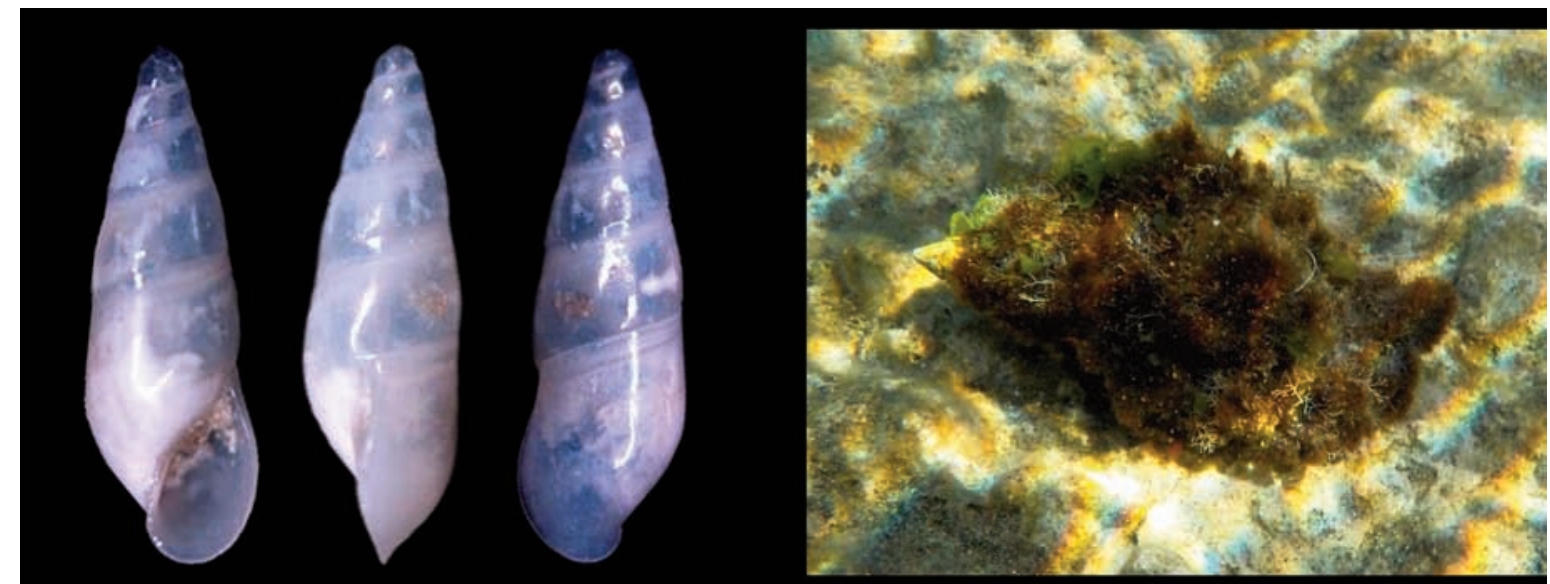

2

3
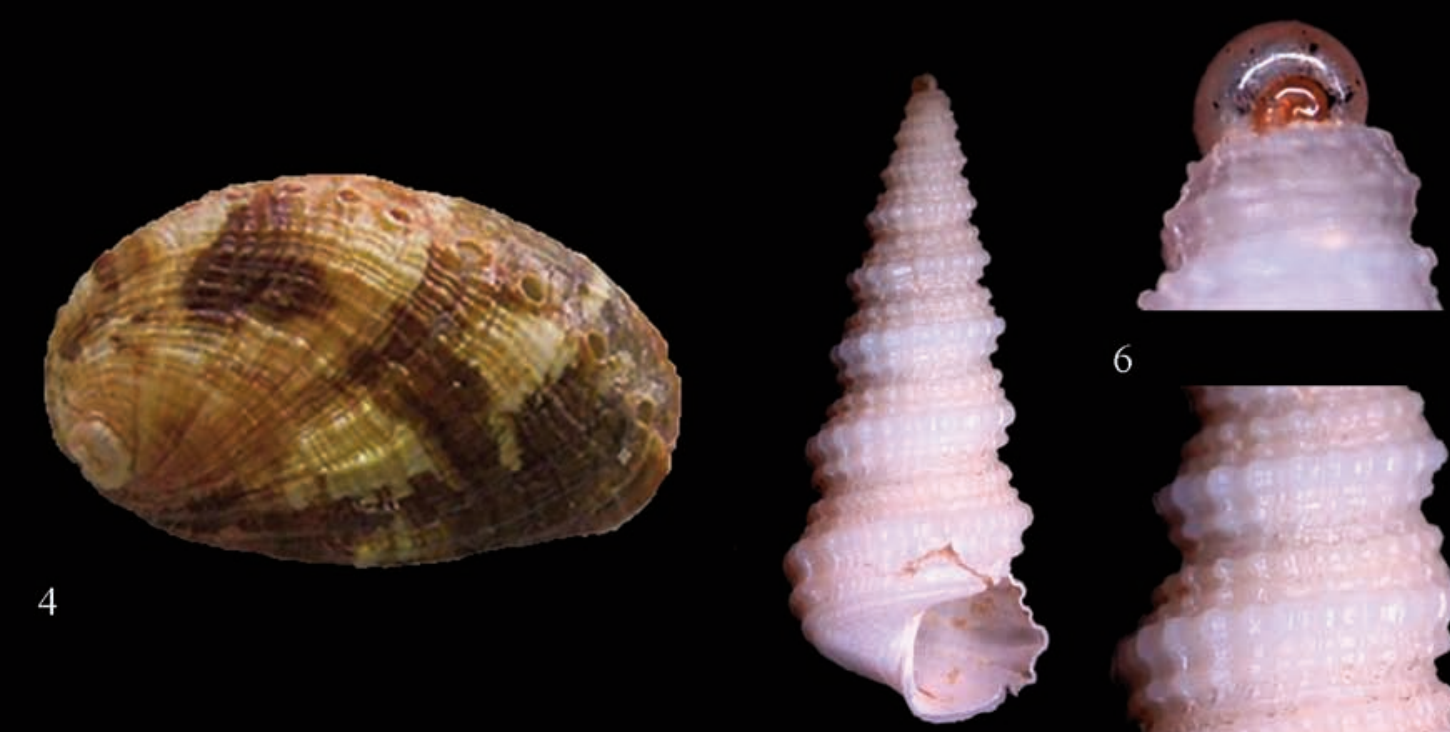

6

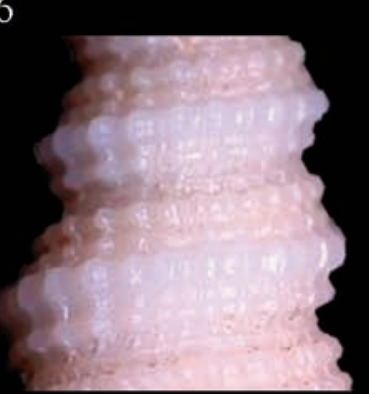

5

7

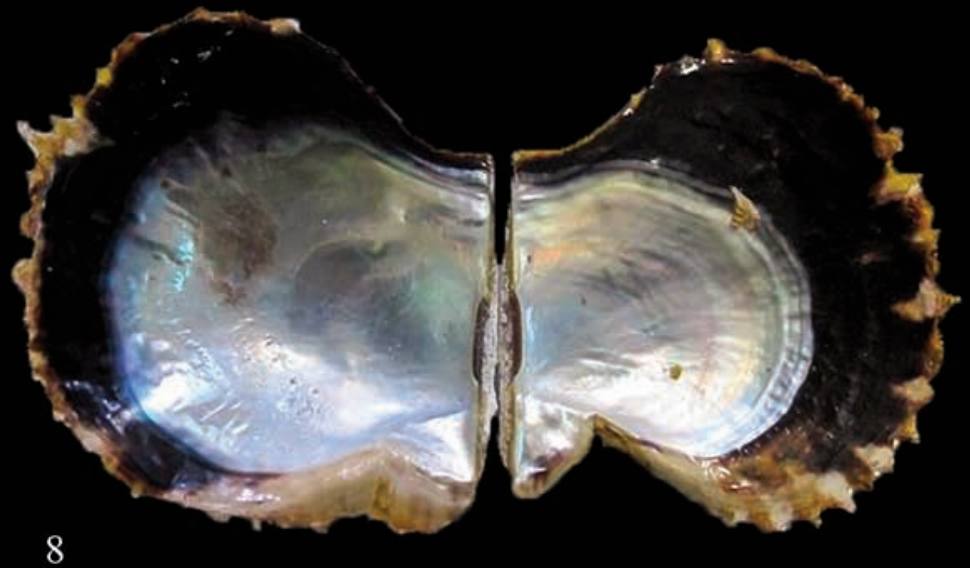

Figure 2. Haliella tyrrenica: ventral, dorsal, and lateral view. Figure 3. In situ living specimen of Monoplex parthenopeus. Figure. 4. Haliotis stomatiaeformis. Figures 5-7. Mathilda bielerei. Fig. 6: apex and protoconcha. Fig. 7: detail of ornamentation. Figure 8. Pinctata imbricata radiata. 


\begin{tabular}{|c|c|c|}
\hline species & site & habitat \\
\hline \multicolumn{3}{|l|}{ POLYPLACOPHORA } \\
\hline Leptochiton cimicoides (Monterosato, 1879) & 3 & PM \\
\hline Leptochiton scabridus (Jeffreys, 1880) & $5 \mathrm{~d}$ & PA \\
\hline Lepidochitona caprearum (Scacchi, 1836) & $5 \mathrm{~d}$ & PA \\
\hline Ischnochiton rissoi (Payaraudeau, 1826) & $35 \mathrm{~d}$ & PA, PM \\
\hline Callochiton septemvalvis (Montagu, 1803) & $5 \mathrm{a}$ & RP \\
\hline Rhyssoplax corallinus (Risso, 1826) & $5 \mathrm{~d}$ & PA \\
\hline Chiton olivaceus Spengler, 1797 & 23 & PA, PM \\
\hline Acanthochitona crinita (Pennat, 1777) & 23 & PA, PM \\
\hline Acanthochitona fascicularis (Linnaeus, 1767) & $35 \mathrm{a}$ & RP, PM \\
\hline \multicolumn{3}{|l|}{ GASTROPODA } \\
\hline Patella caerulea Linnaeus, 1758 & $1235 \mathrm{a}, \mathrm{d}, \mathrm{e}$ & PA, PM, RP \\
\hline Patella ulyssiponensis Gmelin, 1791 & 4 & PM \\
\hline Tectura virginea (O.F. Müller, 1776) & 4 & PM \\
\hline Diodora gibberula (Lamarck, 1822) & 4 & PM \\
\hline Diodora graeca (Linnaeus, 1758 ) & 24 & PA, PM \\
\hline Diodora italica (Defrance, 1820) & 24 & PA, PM \\
\hline Emarginula adriatica O.G. Costa, 1830 & $245 \mathrm{c}$ & PA, PM, CD \\
\hline Emarginula huzardii Payaraudeau, 1826 & $245 \mathrm{c}, \mathrm{d}$ & PA, PM, CD \\
\hline Emarginula octaviana Coen, 1939 & $45 \mathrm{c}$ & PM, CD \\
\hline Emarginula punctulum Piani, 1980 & $5 \mathrm{c}$ & PM \\
\hline Emarginula pustula Thiele in Küster, 1913 & $5 \mathrm{~b}$ & $\mathrm{CD}$ \\
\hline Emarginula rosea Bell, 1824 & $5 \mathrm{c}$ & PM \\
\hline Emarginula tenera Locard, 1891 & $5 \mathrm{~b}, \mathrm{c}$ & $\mathrm{CD}$ \\
\hline Fissurella nubecula (Linnaeus, 1758) & 23 & PA, PM \\
\hline Anatoma aspera (Philippi, 1844) & $5 \mathrm{c}$ & PM \\
\hline Anatoma umbilicata (Jeffreys, 1883) & $5 \mathrm{~d}$ & $\mathrm{CD}$ \\
\hline Scissurella costata d'Orbigny, 1824 & $2345 \mathrm{~d}$ & $\mathrm{PA}, \mathrm{PM}, \mathrm{CD}$ \\
\hline Sinezona cingulata (O.G.Costa, 1861) & $5 c$ & PM \\
\hline Haliotis stomatiaeformis Reeve, 1846 & 3 & PA \\
\hline Haliotis tuberculata tuberculata Linnaeus, 1758 & 234 & AP, PM \\
\hline Lepetella espinosae Dantar \& Luque, 1994 & $5 \mathrm{~b}$ & $\mathrm{CD}$ \\
\hline Clelandella miliaris (Brocchi, 1814) & $5 \mathrm{c}$ & $\mathrm{CD}$ \\
\hline Gibbula ardens (Salis Marschlins, 1793) & 24 & PA \\
\hline Gibbula fanulum (Gmelin, 1791) & $245 c$ & PA, PM, CD \\
\hline Gibbula guttadauri (Philippi, 1836) & 4 & PM \\
\hline Gibbula magus (Linnaeus, 1758) & $45 \mathrm{c}$ & PM, CD \\
\hline Gibbula philberti (Récluz, 1843) & $25 \mathrm{~d}$ & $\mathrm{PA}$ \\
\hline Gibbula racketti (Payraudeau, 1826) & 23 & PA, PM \\
\hline Gibbula turbinoides (Deshayes, 1835) & 34 & PM \\
\hline Steromphala adansonii (Payraudeau, 1826) & $245 \mathrm{a}$ & PA, PM, RP \\
\hline Steromphala divaricata (Linnaeus, 1758) & $2345 \mathrm{a}$ & PA, PM, RP \\
\hline Steromphala rarilineata (Michaud, 1829) & 34 & PM \\
\hline Steromphala umbilicaris (Linnaeus, 1758) & $245 \mathrm{a}$ & PA, RP \\
\hline Steromphala varia (Linnaeus, 1758$)$ & 234 & PA, PM \\
\hline Jujubinus curinii Bogi et Campani, 2006 & $5 \mathrm{~b}$ & $\mathrm{CD}$ \\
\hline
\end{tabular}

Table 1/1. List of the benthic molluses recorded in Capo Milazzo coastal waters. Sites of collection (1-5) and prevalent habitat of each species are indicated: Photophilic Algae (PA); Phanerogam Meadows (PM); Reef Pools (RP); Coastal Detritic and Biodetritic bottoms (CD); Supratidal Rocky reef (SR). 


\begin{tabular}{|c|c|c|}
\hline species & site & habitat \\
\hline Jujubinus exasperatus (Pennat, 1777) & $245 b$ & PA, PM, CD \\
\hline Jujubinus gravinae (Dautzenberg, 1881) & $5 \mathrm{c}$ & $\mathrm{CD}$ \\
\hline Jujubinus montagui (Wood, 1828) & $45 b$ & $\mathrm{PM}, \mathrm{CD}$ \\
\hline Jujubinus striatus (Linnaeus, 1758) & $245 b, c$ & $\mathrm{PA}, \mathrm{PM}, \mathrm{CD}$ \\
\hline Jujubinus tumidulus (Aradas, 1846) & $45 b$ & PM, CD \\
\hline Phorcus articulatus (Lamarck, 1822) & 34 & PM \\
\hline Phorcus mutabilis (Philippi, 1851) & 34 & PM \\
\hline Phorcus richardi (Payraudeau, 1826) & $345 \mathrm{a}$ & PM, RP \\
\hline Phorcus turbinatus (Born, 1778) & $345 a$ & PM, RP \\
\hline Clanculus corallinus (Gmelin, 1791) & $345 \mathrm{~d}$ & PM, PA \\
\hline Clanculus cruciatus (Linnaeus, 1758) & $2345 d$ & PA, PM \\
\hline Clanculus jussieui (Payraudeau, 1826) & $2345 \mathrm{a}, \mathrm{d}$ & PA; PM; RP \\
\hline Calliostoma conulus (Linnaeus, 1758) & $245 b$ & PA, PM, CD \\
\hline Cirsonella romettensis (Granata-Grillo, 1877) & $5 \mathrm{~d}$ & PA \\
\hline Dikoleps depressa (Monterosato, 1880) & $5 \mathrm{~d}$ & PA \\
\hline Dikoleps marianae Rubio, Dantart et Luque, 1998 & $5 \mathrm{~d}$ & PA \\
\hline Skenea catenoides (Monterosato, 1877) & $5 \mathrm{~d}$ & $\mathrm{PA}$ \\
\hline Skenea serpuloides (Montagu, 1808) & $5 \mathrm{~d}$ & PA \\
\hline Lissomphalia bithynoides (Monterosato, 1880 ) & $5 c$ & $\mathrm{CD}$ \\
\hline Skeneoides exilissima (Philippi, 1844) & $5 \mathrm{~d}$ & PA \\
\hline Bolma rugosa (Linnaeus, 1767) & $2345 b$ & PA, PM, CD \\
\hline Danilia tinei (Calcara, 1839$)$ & $5 \mathrm{~d}$ & PA \\
\hline Moelleriopsis messanensis (Seguenza, 1876) & $5 \mathrm{~d}$ & $\mathrm{PA}$ \\
\hline Akritogyra conspicua (Monterosato, 1880 ) & $5 \mathrm{~d}$ & $\mathrm{PA}$ \\
\hline Homalopoma sanguineum (Linnaeus, 1758) & $5 \mathrm{~b}$ & $\mathrm{CD}$ \\
\hline Tricolia cfr. entomocheila Gofas, 1993 & $5 \mathrm{~b}$ & $\mathrm{CD}$ \\
\hline Tricolia pullus pullus (Linnaeus, 1758) & 234 & PA, PM \\
\hline Tricolia punctura Gofas, 1993 & $25 \mathrm{~d}$ & PA \\
\hline Tricolia speciosa (Megerle von Mühlfeld, 1824) & 234 & PA, PM \\
\hline Tricolia tenuis (Michaud, 1829) & 23 & PA, PM \\
\hline Tricolia landini Bogi et Campani, 2007 & 2 & PA \\
\hline Smaragdia viridis (Linnaeus, 1758) & 234 & PA, PM \\
\hline Bittium lacteum (Philippi, 1836) & $2345 d$ & PA, PM \\
\hline Bittium latreillii (Payraudeau, 1826) & $15 \mathrm{c}, \mathrm{d}$ & $\mathrm{PA}, \mathrm{CD}$ \\
\hline Bittium reticulatum (da Costa, 1778 ) & $2345 \mathrm{a}, \mathrm{c}, \mathrm{d}$ & PA, PM, RP, CD \\
\hline Cerithidium submammillatum (De Rayneval et Ponzi, 1854) & $25 \mathrm{~d}$ & PA \\
\hline Cerithium alucastrum (Brocchi, 1814) & $2345 \mathrm{e}$ & PA, PM \\
\hline Cerithium lividulum Risso, 1826 & 23 & $\mathrm{AP}, \mathrm{PM}$ \\
\hline Cerithium protractum Bivona Ant. in Bivona And., 1838 & $45 d$ & PM \\
\hline Cerithium scabridum Philiippi, 1848 & $25 b$ & PA \\
\hline Cerithium vulgatum Bruguière, 1792 & $235 \mathrm{a}, \mathrm{e}$ & PA, PM \\
\hline Fossarus ambiguus (Linnaeus, 1758) & $45 \mathrm{~d}$ & PA, PM \\
\hline Tenagodus obtusus (Schumacher, 1817) & $5 \mathrm{~d}$ & PA \\
\hline Turritella communis Risso, 1826 & $5 \mathrm{~b}$ & $\mathrm{CD}$ \\
\hline Turritella turbona Monterosato, 1877 & 4 & PM \\
\hline Metaxia metaxa (Delle Chiaje, 1828) & $5 b, c$ & $\mathrm{CD}$ \\
\hline
\end{tabular}

Table 1/2. List of the benthic molluscs recorded in Capo Milazzo coastal waters. Sites of collection (1-5) and prevalent habitat of each species are indicated: Photophilic Algae (PA); Phanerogam Meadows (PM); Reef Pools (RP); Coastal Detritic and Biodetritic bottoms (CD); Supratidal Rocky reef (SR). 


\begin{tabular}{|c|c|c|}
\hline species & site & habitat \\
\hline Marshallora adversa (Montagu, 1803) & $25 \mathrm{~d}$ & PA \\
\hline Monophorus erythrosoma (Bouchet et Guillemot, 1978) & $5 e$ & PM \\
\hline Monophorus perversus (Linnaeus, 1758) & $45 b, c$ & $\mathrm{PM}, \mathrm{CD}$ \\
\hline Cheirodonta pallescens (Jeffreys, 1867) & $35 \mathrm{~d}$ & PA, PM \\
\hline Krachia cylindrata (Jeffreys, 1885) & $5 \mathrm{~b}, \mathrm{c}$ & $\mathrm{CD}$ \\
\hline Krachia tiara (Monterosato, 1874 ) & $5 \mathrm{~b}$ & $\mathrm{CD}$ \\
\hline Dizoniopsis concatenata (Conti, 1864) & $5 \mathrm{~b}, \mathrm{e}$ & PM, CD \\
\hline Dizoniopsis coppolae (Aradas, 1870) & $5 \mathrm{~d}$ & PM \\
\hline Cerithiopsis barleei Jeffreys, 1867 & $5 \mathrm{~b}, \mathrm{~d}$ & $\mathrm{PA}, \mathrm{CD}$ \\
\hline Cerithiopsis diadema Monterosato, 1874 & $5 \mathrm{~d}$ & $\mathrm{AP}$ \\
\hline Cerithiopsis fayalensis R.B. Watson, 1886 & 4 & HP \\
\hline Cerithiopsis jeffreysi Watson, 1885 & $5 \mathrm{~d}$ & AP \\
\hline Cerithiopsis minima (Brusina, 1865) & $245 d$ & PA, PM \\
\hline Cerithiopsis scalaris Locard, 1891 & $5 \mathrm{~d}$ & PA \\
\hline Cerithiopsis tubercularis (Montagu, 1803) & $5 \mathrm{~d}$ & PA \\
\hline Opalia coronata (Philippi \& Scacchi, 1840) & $45 \mathrm{c}$ & $\mathrm{PM}, \mathrm{CD}$ \\
\hline Cirsotrema pumiceum (Brocchi, 1814) & 2 & PA \\
\hline Epitonium clathrus (Linnaeus, 1758) & $245 \mathrm{~d}$ & PA, PM \\
\hline Epitonium hispidulum (Monterosato, 1874) & $5 \mathrm{~d}$ & PA \\
\hline Epitonium muricatum (Risso, 1826) & 24 & PA, PM \\
\hline Epitonium pulchellum (Bivona, 1832) & $5 \mathrm{~d}$ & PA \\
\hline Epitonium tiberii (de Boury, 1890) & $45 \mathrm{c}$ & PM, CD \\
\hline Gyroscala lamellosa (Lamarck, 1822) & 3 & PM \\
\hline Acirsa subdecussata (Cantraine, 1835) & $5 \mathrm{c}$ & $\mathrm{CD}$ \\
\hline Aclis ascaris (W. Turton, 1819) & $5 \mathrm{~d}$ & $\mathrm{PA}$ \\
\hline Eulima bilineata Alder, 1848 & $5 \mathrm{~b}$ & $\mathrm{CD}$ \\
\hline Eulima glabra (da Costa, 1778) & $5 \mathrm{~b}$ & $\mathrm{CD}$ \\
\hline Melanella polita (Linnaeus, 1758) & $45 c$ & PM, CD \\
\hline Sabinella bonifaciae (F. Nordsieck, 1974) & $5 \mathrm{~b}, \mathrm{c}$ & $\mathrm{CD}$ \\
\hline Parvioris ibizenca (Nordsiesck, 1968) & $25 \mathrm{c}$ & $\mathrm{PA}, \mathrm{CD}$ \\
\hline Sticteulima jeffreysiana (Brusina, 1869) & $5 \mathrm{c}$ & $\mathrm{CD}$ \\
\hline Vitreolina curva (Monterosato, 1874$)$ & $5 \mathrm{~b}, \mathrm{c}$ & $\mathrm{CD}$ \\
\hline Vitreolina incurva (Bucquoy, Dautzenberg et Dollfus, 1883) & $5 \mathrm{c}$ & $\mathrm{CD}$ \\
\hline Vitreolina perminima (Jeffreys, 1883) & $25 \mathrm{c}$ & $\mathrm{PA}, \mathrm{CD}$ \\
\hline Vitreolina philippi (de Rayneval et Ponzi, 1854) & $25 \mathrm{c}$ & $\mathrm{PA}, \mathrm{CD}$ \\
\hline Nanobalcis nana (Monterosato, 1878) & $5 \mathrm{c}$ & $\mathrm{CD}$ \\
\hline Haliella tyrrhena Di Geronimo et La Perna, 1999 & $5 \mathrm{c}$ & $\mathrm{CD}$ \\
\hline Echinolittorina punctata (Gmelin, 1791) & $125 \mathrm{~d}$ & SR \\
\hline Melarhaphe neritoides (Linnaeus, 1758) & $5 \mathrm{~d}$ & SR \\
\hline Skeneopsis planorbis (O. Fabricius, 1780 ) & $5 \mathrm{c}$ & $\mathrm{CD}$ \\
\hline Eatonina cossurae (Calcara, 1841) & $35 \mathrm{~d}$ & PA, PM \\
\hline Eatonina fulgida (J. Adams, 1797) & $35 \mathrm{~d}$ & PA, PM \\
\hline Eatonina ochroleuca (Brusina, 1869) & $5 \mathrm{~b}$ & $\mathrm{CD}$ \\
\hline Eatonina pumila (Monterosato, 1884) & $5 \mathrm{~d}$ & PA \\
\hline Rissoina bruguieri (Payraudeau, 1826) & 123 & PA, PM \\
\hline Rissoa auriscalpium (Linnaeus, 1758) & 23 & PA, PM \\
\hline
\end{tabular}

Table 1/3. List of the benthic molluscs recorded in Capo Milazzo coastal waters. Sites of collection (1-5) and prevalent habitat of each species are indicated: Photophilic Algae (PA); Phanerogam Meadows (PM); Reef Pools (RP); Coastal Detritic and Biodetritic bottoms (CD); Supratidal Rocky reef (SR). 


\begin{tabular}{|c|c|c|}
\hline species & site & habitat \\
\hline Rissoa decorata Philippi, 1846 & 3 & PM \\
\hline Rissoa guerinii Récluz, 1843 & 23 & PA, PM \\
\hline Rissoa italiensis Verduin, 1985 & $5 \mathrm{~d}$ & PA \\
\hline Rissoa lia (Monterosato, 1884) & $5 \mathrm{a}, \mathrm{d}$ & PA, RP \\
\hline Rissoa membranacea (J. Adams, 1800) & $25 \mathrm{~d}$ & PA \\
\hline Rissoa monodonta Philippi, 1836 & $25 \mathrm{~d}$ & PA \\
\hline Rissoa scurra (Monterosato, 1917) & $5 \mathrm{~d}$ & PA \\
\hline Rissoa similis Scacchi, 1836 & $25 \mathrm{a}, \mathrm{d}$ & PA, RP \\
\hline Rissoa variabilis (Megerle von Mühlfeld, 1824) & $25 \mathrm{a}, \mathrm{c}$ & PA, RP, CD \\
\hline Rissoa ventricosa Desmarest, 1814 & 23 & PA, PM \\
\hline Rissoa violacea Desmarest, 1814 & $35 \mathrm{~d}$ & PM, PA \\
\hline Alvania beanii (Hanley in Thorpe, 1844) & 4 & PM \\
\hline Alvania cancellata (da Costa, 1778) & $45 \mathrm{a}, \mathrm{d}$ & PM, RP, PA \\
\hline Alvania cimex (Linnaeus, 1758) & $245 \mathrm{a}, \mathrm{d}$ & PA, PM, RP \\
\hline Alvania cimicoides (Forbes, 1844) & $5 c$ & $\mathrm{CD}$ \\
\hline Alvania clathrella L. Seguenza, 1903 & $5 e$ & $\mathrm{CD}$ \\
\hline Alvania dictyophora (Philippi, 1844) & 5e & $\mathrm{CD}$ \\
\hline Alvania discors (T. Allan, 1818) & 234 & $\mathrm{PA}, \mathrm{PM}$ \\
\hline Alvania gagliniae Amati, 1985 & $5 c$ & $\mathrm{CD}$ \\
\hline Alvania geryonia (Nardo, 1847) & $45 \mathrm{a}$ & PM, RP \\
\hline Alvania hallgassi Amati et Oliverio, 1985 & $5 \mathrm{~d}$ & PA \\
\hline Alvania hirta (Monterosato, 1884) & $25 b$ & $\mathrm{PA}, \mathrm{CD}$ \\
\hline Alvania hispidula (Monterosato, 1884 ) & $5 c$ & $\mathrm{CD}$ \\
\hline Alvania lanciae (Calcara, 1845) & $2345 a$ & PA, PM, RP \\
\hline Alvania lineata Risso, 1826 & $2345 \mathrm{~b}$ & $\mathrm{PA}, \mathrm{PM}, \mathrm{CD}$ \\
\hline Alvania pagodula (Bucquoy, Dautzenberg et Dollfus, 1884) & $5 \mathrm{~b}, \mathrm{c}$ & $\mathrm{CD}$ \\
\hline Alvania punctura (Montagu, 1803) & $5 b, c$ & $\mathrm{CD}$ \\
\hline Alvania rudis (Philippi, 1844) & $5 b, c$ & $\mathrm{CD}$ \\
\hline Alvania scabra (Philippi, 1844) & $5 a, b, c$ & $\mathrm{RP}, \mathrm{CD}$ \\
\hline Alvania subcrenulata (Bucquoy, Dautzenberg et Dollfus, 1884) & $5 \mathrm{~b}$ & $\mathrm{CD}$ \\
\hline Alvania testae (Aradas et Maggiore, 1844) & $5 \mathrm{~d}$ & $\mathrm{CD}$ \\
\hline Alvania weinkauffi jacobusi Oliverio, Amati et Nofroni, 1986 & $5 \mathrm{~d}$ & $\mathrm{CD}$ \\
\hline Alvania zetlandica (Montagu, 1815) & $5 \mathrm{c}$ & $\mathrm{CD}$ \\
\hline Crisilla beniamina (Monterosato, 1884 ) & $5 b$ & $\mathrm{CD}$ \\
\hline Crisilla semistriata (Montagu, 1808) & $5 \mathrm{~b}$ & $\mathrm{CD}$ \\
\hline Manzonia crassa (Kanmacher, 1798) & $25 \mathrm{c}$ & $\mathrm{RP}, \mathrm{CD}$ \\
\hline Obtusella intersecta (S. Wood, 1857) & $5 c$ & $\mathrm{CD}$ \\
\hline Obtusella macilenta (Monterosato, 1880) & $5 c$ & $\mathrm{CD}$ \\
\hline Botryphallus epidauricus (Brusina, 1866) & $5 \mathrm{c}$ & $\mathrm{CD}$ \\
\hline Peringiella elegans (Locard, 1891) & 1 & $\mathrm{CD}$ \\
\hline Pusillina inconspicua (Alder, 1844) & $45 c$ & PM, CD \\
\hline Pusillina lineolata (Michaud, 1830) & $25 \mathrm{a} 5 \mathrm{~b}$ & $\mathrm{AP}, \mathrm{RP}, \mathrm{CD}$ \\
\hline Pusillina marginata (Michaud, 1830) & 2 & PA \\
\hline Pusillina philippi (Aradas et Maggiore, 1844) & 3 & PM \\
\hline Pusillina radiata (Philippi, 1836) & 4 & PM \\
\hline Setia amabilis (Locard, 1886) & $5 \mathrm{a}$ & RP \\
\hline
\end{tabular}

Table 1/4. List of the benthic molluscs recorded in Capo Milazzo coastal waters. Sites of collection (1-5) and prevalent habitat of each species are indicated: Photophilic Algae (PA); Phanerogam Meadows (PM); Reef Pools (RP); Coastal Detritic and Biodetritic bottoms (CD); Supratidal Rocky reef (SR). 


\begin{tabular}{|c|c|c|}
\hline species & site & habitat \\
\hline Setia scillae (Aradas et Benoit, 1876) & 2 & PA \\
\hline Pisinna glabrata (Megerle von Mühlfeld, 1824) & 3 & PM \\
\hline Nodulus contortus (Jeffreys, 1856) & $5 b$ & $\mathrm{CD}$ \\
\hline Paludinella globularis (Hanley in Thorpe, 1844) & $5 \mathrm{a}$ & RP \\
\hline Barleeia unifasciata (Montagu, 1803) & $235 \mathrm{a}$ & PA, PM, RP \\
\hline Caecum auriculatum De Folin, 1868 & $25 d$ & PA, PM \\
\hline Caecum clarkii Carpenter, 1859 & 4 & PM \\
\hline Caecum subannulatum De Folin, 1870 & $25 \mathrm{c}$ & $\mathrm{CD}$ \\
\hline Caecum trachea (Montagu, 1803) & $145 \mathrm{~d}$ & PA, PM \\
\hline Hyala vitrea (Montagu, 1803) & 23 & $\mathrm{PA}, \mathrm{PM}$ \\
\hline Tornus subcarinatus (Montagu, 1803) & $5 \mathrm{~d}$ & PA \\
\hline Circulus striatus (Philippi, 1836) & $5 b$ & CD \\
\hline Truncatella subcylindrica (Linnaeus, 1767) & 234 & PA, PM \\
\hline Thylacodes arenarius (Linnaeus, 1758) & 3 & PM \\
\hline Dendropoma cristatum (Biondi, 1859) & $235 d$ & PA, PM, \\
\hline Petaloconchus glomeratus (Linnaeus, 1758) & 3 & PM \\
\hline Thylaeodus rugulosus (Monterosato, 1878) & 2 & PA \\
\hline Aporrhais pespelecani (Linnaeus, 1758) & $345 \mathrm{c}$ & PM, CD \\
\hline Crepidula unguiformis Lamarck, 1822 & $2345 b$ & PA, PM, CD \\
\hline Calyptraea chinensis (Linnaeus, 1758) & $145 \mathrm{~b}$ & PA, PM, CD \\
\hline Capulus ungaricus (Linnaeus, 1758) & $45 \mathrm{c}$ & PM, CD \\
\hline Lamellaria perspicua (Linnaeus, 1758) & $35 \mathrm{~d}$ & PA, PM \\
\hline Erato voluta (Montagu, 1803) & $5 \mathrm{~d}$ & PA \\
\hline Trivia arctica (Pulteney, 1799) & $1245 \mathrm{c}$ & $\mathrm{PA}, \mathrm{PM}, \mathrm{CD}$ \\
\hline Trivia mediterranea (Risso, 1826) & 14 & PM \\
\hline Trivia monacha (da Costa, 1778$)$ & 4 & PM \\
\hline Luria lurida (Linnaeus, 1758) & 23 & $\mathrm{AP}, \mathrm{PM}$ \\
\hline Naria spurca (Linnaeus, 1758) & 3 & PM \\
\hline Simnia spelta (Linnaeus, 1758) & $5 \mathrm{~b}$ & $\mathrm{CD}$ \\
\hline Pseudosimnia carnea (Poiret, 1789) & $5 \mathrm{~b}$ & $\mathrm{CD}$ \\
\hline Naticarius stercusmuscarum (Gmelin, 1791) & $2345 \mathrm{c}$ & PM, CD \\
\hline Naticarius hebraeus (Martyn, 1786) & $345 \mathrm{c}$ & PM, CD \\
\hline Notocochlis dillwynii Payaraudeau, 1826 & 3 & PM \\
\hline Tectonatica sagraiana (d'Orbigny, 1842) & 23 & PM, CD \\
\hline Euspira fusca (Blainville, 1825) & $5 b$ & $\mathrm{CD}$ \\
\hline Euspira intricata (Donovan, 1804) & 234 & PM, CD \\
\hline Euspira macilenta (Philippi, 1844) & 4 & PM \\
\hline Euspira nitida (Donovan, 1804) & 14 & PM \\
\hline Galeodea echinophora (Linnaeus, 1758) & 2 & $\mathrm{CD}$ \\
\hline Neverita josephinia Risso, 1826 & 23 & PM \\
\hline Semicassis granulata (Born, 1778) & 3 & PM \\
\hline Ranella olearium (Linnaeus, 1758) & $5 \mathrm{c}$ & $\mathrm{CD}$ \\
\hline Charonia lampas (Linnaeus, 1758) & $5 c$ & $\mathrm{CD}$ \\
\hline Monoplex corrugatus (Lamarck, 1816) & 3 & PM \\
\hline Monoplex parthenopeus (Salis Marschlins, 1793) & $5 \mathrm{c}$ & $\mathrm{CD}$ \\
\hline Cabestana cutacea (Linnaeus, 1767) & 4 & PM \\
\hline
\end{tabular}

Table 1/5. List of the benthic molluscs recorded in Capo Milazzo coastal waters. Sites of collection (1-5) and prevalent habitat of each species are indicated: Photophilic Algae (PA); Phanerogam Meadows (PM); Reef Pools (RP); Coastal Detritic and Biodetritic bottoms (CD); Supratidal Rocky reef (SR). 


\begin{tabular}{|c|c|c|}
\hline species & site & habitat \\
\hline Bursa scrobilator (Linnaeus, 1758) & $5 \mathrm{~d}$ & PA \\
\hline Hexaplex trunculus (Linnaeus, 1758) & $2345 \mathrm{c}$ & $\mathrm{PA}, \mathrm{PM}, \mathrm{CD}$ \\
\hline Dermomurex scalaroides (Blanville, 1829) & $45 \mathrm{a}$ & PM, RP \\
\hline Ocenebra edwardsii (Payraudeau, 1826) & 3 & PM \\
\hline Ocenebra erinaceus (Linnaeus, 1758) & 4 & $\mathrm{PM}$ \\
\hline Ocinebrina aciculata (Lamarck, 1822) & $245 b, c$ & $\mathrm{PA}, \mathrm{PM}, \mathrm{CD}$ \\
\hline Hadriania craticulata Bucquoy et Dautzenberg, 1882 & $25 \mathrm{c}$ & $\mathrm{CD}$ \\
\hline Murexsul aradasii (Monterosato in Poirier, 1883) & $5 b, c$ & $\mathrm{CD}$ \\
\hline Muricopsis cristata (Brocchi, 1814) & $25 \mathrm{~d}$ & PA \\
\hline Typhinellus labiatus (de Cristofori et Jan,1832) & 23 & $\mathrm{PA}, \mathrm{PM}$ \\
\hline Pagodula echinata (Kiener, 1840) & $5 \mathrm{c}$ & $\mathrm{CD}$ \\
\hline Trophonopsis barvicensis (Johnston, 1825) & $5 \mathrm{c}$ & $\mathrm{CD}$ \\
\hline Stramonita haemastoma (Linnaeus, 1767) & 1234 & PA, PM \\
\hline Coralliophila brevis (Blainville, 1832) & $5 c, d$ & $\mathrm{CD}, \mathrm{PM}$ \\
\hline Coralliophila meyendorffii (Calcara, 1845) & 3 & PA \\
\hline Hirtomurex squamosus (Bivona Ant. in Bivona And., 1838) & $5 \mathrm{~d}$ & PA \\
\hline Volvarina mitrella (Risso, 1826) & $45 \mathrm{~d}$ & PM \\
\hline Granulina marginata (Bivona, 1832) & $25 \mathrm{a}, \mathrm{d}$ & PA, PM, RP \\
\hline Granulina mediterranea Landau, La Perna et Marquet, 2006 & 4 & PM \\
\hline Granulina occulta (Monterosato, 1896) & $5 \mathrm{~b}$ & $\mathrm{CD}$ \\
\hline Gibberula miliaria (Linnaeus, 1758) & $2345 d$ & PA, PM \\
\hline Gibberula philippi (Monterosato, 1878) & $245 a, d$ & PN, PA, RP \\
\hline Gibberula recondita Monterosato, 1884 & $25 \mathrm{~d}$ & PA \\
\hline Isara cornea (Lamarck,1811) & $35 \mathrm{~d}$ & PA, PM \\
\hline Episcomitra cornicula (Linnaeus, 1758 ) & $245 b$ & PA, PM, CD \\
\hline Pusia ebenus (Lamarck, 1811) & 24 & PA, PM \\
\hline Pusia savignyi (Payraudeau, 1826) & $235 \mathrm{~d}$ & PA, PM \\
\hline Pusia tricolor $($ Gmelin, 1791$)$ & $245 \mathrm{a}, \mathrm{d}$ & PA, PM, RP \\
\hline Euthria cornea (Linnaeus, 1758) & 24 & PA, PM \\
\hline Pisania striata (Gmelin, 1791) & 24 & PA, PM \\
\hline Chauvetia lefebvrii (Maravigna, 1840) & $5 b, c$ & $\mathrm{CD}$ \\
\hline Chauvetia mamillata (Risso, 1826) & $45 b, c$ & PM, CD \\
\hline Chauvetia procerula (Monterosato, 1889) & $5 \mathrm{~b}, \mathrm{c}$ & $\mathrm{CD}$ \\
\hline Chauvetia recondita (Brugnone, 1873) & $5 \mathrm{~d}$ & PA \\
\hline Chauvetia turritellata (Deshayes, 1835) & $5 b, c$ & $\mathrm{CD}$ \\
\hline Aplus dorbignyi (Payraudeau, 1826) & 23 & PA, PM \\
\hline Cumia reticulata (Blainville, 1829) & $45 b$ & PM, CD \\
\hline Tritia corniculum (Olivi, 1792) & $5 \mathrm{~d}$ & PA \\
\hline Tritia cuvierii (Payraudeau, 1826) & 234 & PA, PM \\
\hline Tritia lima (Dillwyn, 1817) & $45 b, c$ & PM, CD \\
\hline Tritia mutabilis (Linnaeus, 1758) & 2 & PA \\
\hline Tritia neritea (Linnaeus, 1758) & 23 & PA, PM \\
\hline Tritia pygmaea (Lamarck, 1822) & 23 & PA, PM \\
\hline Columbella rustica (Linnaeus, 1758) & $2345 \mathrm{a}$ & PA, PM, RP \\
\hline Mitrella coccinea (Philippi, 1836) & $5 \mathrm{~d}$ & PA \\
\hline Mitrella scripta (Linnaeus, 1758) & $25 \mathrm{a}, \mathrm{b}, \mathrm{c}$ & PA, RP, CD \\
\hline
\end{tabular}

Table 1/6. List of the benthic molluscs recorded in Capo Milazzo coastal waters. Sites of collection (1-5) and prevalent habitat of each species are indicated: Photophilic Algae (PA); Phanerogam Meadows (PM); Reef Pools (RP); Coastal Detritic and Biodetritic bottoms (CD); Supratidal Rocky reef (SR). 


\begin{tabular}{|c|c|c|}
\hline species & site & habitat \\
\hline Tarantinaea lignaria (Linnaeus, 1758) & 4 & PM \\
\hline Aptyxis syracusana (Linnaeus, 1758 ) & 3 & PM \\
\hline Fusinus dimassai Buzzurro et Russo, 2007 & $5 c$ & $\mathrm{CD}$ \\
\hline Fusinus parvulus (Monterosato, 1884) & $5 \mathrm{~b}$ & $\mathrm{CD}$ \\
\hline Fusinus pulchellus (Philippi, 1840) & $45 \mathrm{c}$ & $\mathrm{PM}, \mathrm{CD}$ \\
\hline Fusinus rostratus (Olivi, 1792) & $5 \mathrm{c}$ & $\mathrm{CD}$ \\
\hline Crassopleura maravignae (Bivona Ant. in Bivona And., 1838) & $5 \mathrm{c}$ & $\mathrm{CD}$ \\
\hline Haedropleura septangularis (Montagu, 1803) & $25 \mathrm{~b}$ & $\mathrm{PA}, \mathrm{CD}$ \\
\hline Drilliola emendata (Monterosato, 1872) & $5 \mathrm{c}$ & $\mathrm{CD}$ \\
\hline Drilliola loprestiana (Calcara, 1841) & $5 \mathrm{c}$ & $\mathrm{CD}$ \\
\hline Mitromorpha olivoidea (Cantraine, 1835) & $245 d$ & $\mathrm{PA}, \mathrm{PM}$ \\
\hline Clathromangelia granum (Philippi, 1844) & $45 \mathrm{a}, \mathrm{d}$ & PM, RP, PA \\
\hline Comarmondia gracilis (Montagu, 1803) & $45 \mathrm{c}$ & PM, CD \\
\hline Conus ventricosus Gmelin, 1791 & 234 & PA, PM \\
\hline Raphitoma atropurpurea (Locard et Caziot, 1900) & $5 \mathrm{c}$ & $\mathrm{CD}$ \\
\hline Raphitoma concinna (Scacchi, 1836) & $245 d$ & PA, PM \\
\hline Raphitoma echinata (Brocchi, 1814) & $25 \mathrm{c}$ & $\mathrm{PA}, \mathrm{CD}$ \\
\hline Raphitoma laviae (Philippi, 1844) & $45 \mathrm{c}$ & $\mathrm{PM}, \mathrm{CD}$ \\
\hline Raphitoma leufroyi (Michaud, 1828) & $5 \mathrm{~d}$ & PA \\
\hline Raphitoma linearis (Montagu, 1803) & $245 \mathrm{c}$ & $\mathrm{PA}, \mathrm{PM}, \mathrm{CD}$ \\
\hline Raphitoma lineolata (Bucquoy, Dautzenberg et Dollfus, 1883) & $25 \mathrm{c}$ & $\mathrm{AP}, \mathrm{CD}$ \\
\hline Teretia teres $($ Reeve, 1844$)$ & $5 b, c$ & $\mathrm{CD}$ \\
\hline Sorgenfreispira brachystoma (Philippi, 1844) & $245 \mathrm{c}$ & PA, PM,CD \\
\hline Bela decussata (Locard, 1891) & $5 c$ & $\mathrm{CD}$ \\
\hline Bela menkhorsti van Aarten, 1988 & $25 \mathrm{c}$ & $\mathrm{PA}, \mathrm{CD}$ \\
\hline Bela nebula (Montagu, 1803) & 124 & PA, PM \\
\hline Bela zenetouae (van Aarten, 1988) & $5 \mathrm{c}$ & $\mathrm{CD}$ \\
\hline Mangelia attenuata (Montagu, 1803) & $245 b$ & $\mathrm{PA}, \mathrm{PM}, \mathrm{CD}$ \\
\hline Mangelia costata (Pennant, 1777) & $25 \mathrm{~b}, \mathrm{c}$ & $\mathrm{PA}, \mathrm{CD}$ \\
\hline Mangelia costulata Risso, 1826 & $245 b$ & $\mathrm{PA}, \mathrm{PM}, \mathrm{CD}$ \\
\hline Mangelia multilineolata (Deshayes, 1835) & $45 b, c$ & $\mathrm{PM}, \mathrm{CD}$ \\
\hline Mangelia paciniana (Calcara, 1839) & $5 \mathrm{~b}$ & $\mathrm{CD}$ \\
\hline Mangelia striolata Risso, 1826 & $25 \mathrm{~b}$ & $\mathrm{PA}, \mathrm{CD}$ \\
\hline Mangelia taeniata (Deshayes, 1835) & $245 b$ & $\mathrm{PA}, \mathrm{PM}, \mathrm{CD}$ \\
\hline Mangelia unifasciata (Deshayes, 1835) & $245 \mathrm{~d}$ & PA, PM, CD \\
\hline Mangelia vauquelini (Payraudeau, 1826) & $5 \mathrm{~d}$ & PA \\
\hline Basisulcata lepida (Bayer, 1942) & $5 c$ & $\mathrm{CD}$ \\
\hline Philippia hybrida (Linnaeus, 1758) & $5 c$ & $\mathrm{CD}$ \\
\hline Discotectonica discus (Philippi, 1844) & $5 \mathrm{c}$ & $\mathrm{CD}$ \\
\hline Solatisonax alleryi (Seguenza G., 1876) & $5 c$ & CD \\
\hline Heliacus fallaciosus (Tiberi, 1872) & $5 c$ & $\mathrm{CD}$ \\
\hline Pseudotorinia architae (O.G. Costa,1841) & $5 \mathrm{c}$ & $\mathrm{CD}$ \\
\hline Mathilda bieleri Smriglio et Mariottini, 2007 & $5 \mathrm{c}$ & $\mathrm{CD}$ \\
\hline Mathilda cochlaeformis Brugnone, 1873 & $5 c$ & $\mathrm{CD}$ \\
\hline Rissoella inflata (Monterosato, 1880) & $25 \mathrm{c}$ & $\mathrm{PA}, \mathrm{CD}$ \\
\hline Rissoella opalina (Jeffreys, 1848) & $25 \mathrm{c}$ & $\mathrm{PA}, \mathrm{CD}$ \\
\hline
\end{tabular}

Table 1/7. List of the benthic molluscs recorded in Capo Milazzo coastal waters. Sites of collection (1-5) and prevalent habitat of each species are indicated: Photophilic Algae (PA); Phanerogam Meadows (PM); Reef Pools (RP); Coastal Detritic and Biodetritic bottoms (CD); Supratidal Rocky reef (SR). 


\begin{tabular}{|c|c|c|}
\hline species & site & habitat \\
\hline Ammonicera fischeriana (Monterosato, 1869) & $25 \mathrm{c}$ & $\mathrm{PA}, \mathrm{CD}$ \\
\hline Ammonicera rota (Forbes et Hanley, 1850) & $25 \mathrm{c}$ & $\mathrm{PA}, \mathrm{CD}$ \\
\hline Omalogyra atomus (Philippi, 1841) & $25 \mathrm{c}$ & $\mathrm{PA}, \mathrm{CD}$ \\
\hline Omalogyra simplex (Costa O.G., 1861) & $25 \mathrm{c}$ & $\mathrm{PA}, \mathrm{CD}$ \\
\hline Retrotortina fuscata Chaster, 1896 & $5 c$ & $\mathrm{CD}$ \\
\hline Tomura depressa (Granata-Grillo, 1877) & $25 \mathrm{c}$ & $\mathrm{PA}, \mathrm{CD}$ \\
\hline Xenoskenea pellucida (Monterosato, 1874) & $25 \mathrm{c}$ & $\mathrm{PA}, \mathrm{CD}$ \\
\hline Odostomia acuta Jeffreys, 1848 & $25 b$ & $\mathrm{PA}, \mathrm{CD}$ \\
\hline Odostomia suboblonga Jeffreys, 1884 & $5 \mathrm{~d}$ & PA \\
\hline Odostomia turrita Hanley, 1844 & $5 \mathrm{~d}$ & $\mathrm{PA}$ \\
\hline Brachystomia scalaris MacGillivray, 1843 & $25 b$ & $\mathrm{PA}, \mathrm{CD}$ \\
\hline Megastomia alungata (Nordiesck, 1972) & $25 d$ & PA \\
\hline Megastomia conoidea (Brocchi, 1814) & $25 a, d$ & PA, RP \\
\hline Euparthenia humboldti (Risso, 1826) & $45 b, d$ & PM, CD \\
\hline Folinella excavata (Phillippi, 1836) & $245 b$ & $\mathrm{PA}, \mathrm{PM}, \mathrm{CD}$ \\
\hline Ondina crystallina Locard, 1891 & 2 & PA \\
\hline Ondina vitrea (Brusina, 1866) & $25 \mathrm{~d}$ & $\mathrm{PA}$ \\
\hline Ondina warreni (Thompson, 1845) & $5 d$ & $\mathrm{PA}$ \\
\hline Chrysallida stefanisi (Jeffreys, 1869) & $5 b$ & $\mathrm{CD}$ \\
\hline Parthenina clathrata (Jeffreys, 1848) & $5 b$ & $\mathrm{CD}$ \\
\hline Parthenina decussata (Montagu, 1803) & $5 \mathrm{~d}$ & PA \\
\hline Parthenina emaciata (Brusina, 1866) & $25 b, d$ & $\mathrm{PA}, \mathrm{CD}$ \\
\hline Parthenina interstincta (J. Adams, 1797) & $25 b$ & $\mathrm{PA}, \mathrm{CD}$ \\
\hline Parthenina monozona (Brusina, 1869) & $245 b$ & PA, PM, CD \\
\hline Tragula fenestrata (Jeffreys, 1848) & $25 \mathrm{~d}$ & PA \\
\hline Odostomella bicincta (Tiberi, 1868) & $5 \mathrm{~d}$ & PA \\
\hline Odostomella doliolum (Philippi, 1844) & $235 b$ & PA, PM, CD \\
\hline Turbonilla acuta (Donovan, 1804) & $25 b$ & PA, CD \\
\hline Turbonilla acutissima Monterosato, 1884 & $25 b$ & $\mathrm{PA}, \mathrm{CD}$ \\
\hline Turbonilla edgari (Melvill, 1896) & $5 \mathrm{~b}$ & $\mathrm{CD}$ \\
\hline Turbonilla gradata Bucquoy, Dautzenberg et Dollfus, 1883 & $25 b$ & $\mathrm{PA}, \mathrm{CD}$ \\
\hline Turbonilla lactea (Linnaeus, 1758 ) & $245 b, c$ & PA, PM, CD \\
\hline Turbonilla pusilla (Philippi, 1844) & $5 b$ & $\mathrm{CD}$ \\
\hline Pyrgiscus jeffreysii (Jeffreys, 1848) & $245 b$ & $\mathrm{PA}, \mathrm{PM}, \mathrm{CD}$ \\
\hline Pyrgiscus rufus (Philippi, 1836) & $245 b$ & $\mathrm{PA}, \mathrm{PM}, \mathrm{CD}$ \\
\hline Pyrgostylus striatulus (Linnaeus, 1758) & $245 b$ & PA, PM, CD \\
\hline Eulimella acicula (Philippi, 1836) & $25 b, d$ & $\mathrm{PA}, \mathrm{CD}$ \\
\hline Eulimella cerulli (Cossmann, 1916) & $25 b, d$ & $\mathrm{PA}, \mathrm{CD}$ \\
\hline Eulimella scillae (Scacchi, 1835) & $45 b, d$ & $\mathrm{PA}, \mathrm{CD}$ \\
\hline Eulimella ventricosa (Forbes, 1844) & $25 b, d$ & $\mathrm{PA}, \mathrm{CD}$ \\
\hline Clathrella clathrata (Philippi, 1844) & $5 \mathrm{~b}$ & CD \\
\hline Ebala nitidissima (Montagu, 1803) & $5 b$ & $\mathrm{CD}$ \\
\hline Ebala pointeli (de Folin, 1868 ) & $45 b$ & PM, CD \\
\hline Cima minima (Jeffreys, 1858) & $25 b$ & $\mathrm{PA}, \mathrm{CD}$ \\
\hline Ringicula auriculata (Ménard de la Groye, 1811) & $25 b, c$ & PA, CD \\
\hline Ringicula conformis Monterosato, 1877 & $25 b$ & PA. CD \\
\hline
\end{tabular}

Table 1/8. List of the benthic molluscs recorded in Capo Milazzo coastal waters. Sites of collection (1-5) and prevalent habitat of each species are indicated: Photophilic Algae (PA); Phanerogam Meadows (PM); Reef Pools (RP); Coastal Detritic and Biodetritic bottoms (CD); Supratidal Rocky reef (SR). 


\begin{tabular}{|c|c|c|}
\hline $\begin{array}{ll} & \text { species } \\
\end{array}$ & site & habitat \\
\hline Bulla striata Bruguière, 1792 & 23 & PA, PM \\
\hline Retusa crebrisculpta (Monterosato, 1884) & $25 b$ & $\mathrm{PA}, \mathrm{CD}$ \\
\hline Retusa leptoeneilema (Brusina, 1866) & $125 \mathrm{c}$ & PA,CD \\
\hline Retusa mammillata (Philippi, 1836) & $15 \mathrm{~b}$ & $\mathrm{PA}, \mathrm{CD}$ \\
\hline Retusa minutissima (Monterosato, 1878) & $25 b$ & $\mathrm{PA}, \mathrm{CD}$ \\
\hline Retusa truncatula (Bruguière, 1792) & $245 b$ & PA, PM, CD \\
\hline Retusa umbilicata (Montagu, 1803) & $25 b$ & $\mathrm{PA}, \mathrm{CD}$ \\
\hline Pyrunculus hoernesi (Weinkauff, 1866) & $5 \mathrm{~b}, \mathrm{c}$ & $\mathrm{CD}$ \\
\hline Volvulella acuminata (Bruguière, 1792) & $245 b, c$ & PA, PM, CD \\
\hline Hатіпоеа sp. & $245 b$ & PA, PM, CD \\
\hline Atys jeffreysi (Weinkauff, 1866) & $45 \mathrm{c}$ & PM, CD \\
\hline Weinkauffia turgidula (Forbes, 1844) & $25 \mathrm{c}$ & $\mathrm{PA}, \mathrm{CD}$ \\
\hline Hermania scabra (O.F. Müller, 1784) & $245 \mathrm{c}$ & $\mathrm{PA}, \mathrm{PM}, \mathrm{CD}$ \\
\hline Philine angulata Jeffreys, 1867 & $5 \mathrm{~b}$ & $\mathrm{CD}$ \\
\hline Philine catena (Montagu, 1803) & $45 \mathrm{c}$ & $\mathrm{PM}, \mathrm{CD}$ \\
\hline Philine denticulata (J. Adams, 1800) & $5 \mathrm{~d}$ & PA \\
\hline Philine intricata Monterosato, 1884 & $5 b, d$ & $\mathrm{CD}, \mathrm{PA}$ \\
\hline Philine sp. & $5 \mathrm{c}$ & $\mathrm{CD}$ \\
\hline Philine punctata (J. Adams, 1800) & $5 \mathrm{c}$ & $\mathrm{CD}$ \\
\hline Philine quadripartita Ascanius, 1772 & 1 & PA \\
\hline Philine striatula Monterosato, 1874 & $25 \mathrm{c}$ & $\mathrm{PA}, \mathrm{CD}$ \\
\hline Scaphander lignarius (Linnaeus, 1758) & 4 & PM \\
\hline Roxania utriculus (Brocchi, 1814) & $245 c$ & PA, PM, CD \\
\hline Cylichna cylindracea (Pennat, 1777) & $5 b, c$ & $\mathrm{CD}$ \\
\hline Umbraculum umbraculum (Lightfoot, 1786) & $5 c$ & $\mathrm{CD}$ \\
\hline Tylodina perversa (Gmelin, 1791) & $5 d$ & $\mathrm{PA}$ \\
\hline Berthella aurantiaca (Risso, 1818) & $5 \mathrm{~d}$ & $\mathrm{PA}$ \\
\hline Berthella plumula (Montagu, 1803) & $5 \mathrm{~d}$ & $\mathrm{PA}$ \\
\hline Berthella stellata (Risso, 1826) & $5 \mathrm{~d}$ & PA \\
\hline Aplysia dactylomela Rang, 1828 & $5 e$ & PM \\
\hline Petalifera petalifera (Rang, 1828) & 2 & PA \\
\hline Notarchus punctatus Philippi, 1836 & $45 b$ & PM, CD \\
\hline Williamia gussoni (Costa O.G., 1829) & $45 b$ & $\mathrm{PM}, \mathrm{CD}$ \\
\hline \multicolumn{3}{|l|}{ BIVALVIA } \\
\hline Nucula nitidosa Winckworth, 1930 & 12 & PA \\
\hline Nucula nucleus (Linnaeus, 1758) & 4 & PM \\
\hline Nucula sulcata Bronn, 1831 & $245 b, c, d$ & PA, PM, CD \\
\hline Lembulus pella (Linnaeus, 1758) & $12345 b$ & PA, PM, CD \\
\hline Saccella commutata (Philippi, 1844) & $45 b$ & PM, CD \\
\hline Yoldiella philippiana (Nyst, 1845) & $5 \mathrm{~b} 5 \mathrm{c}$ & $\mathrm{CD}$ \\
\hline Arca noae Linnaeus, 1758 & $12345 \mathrm{a}, \mathrm{b}$ & $\mathrm{PA}, \mathrm{PM}, \mathrm{RP}, \mathrm{CL}$ \\
\hline Arca tetragona Poli, 1795 & $145 \mathrm{~b}, \mathrm{c}$ & PA, PM, CD \\
\hline Asperarca nodulosa (O F. Müller, 1776) & $45 b, c$ & PM, CD \\
\hline Asperarca secreta La Perna, 1998 & $25 b, c, d$ & $\mathrm{PA}, \mathrm{CD}$ \\
\hline Barbatia barbata (Linnaeus, 1758) & 1234 & PA, PM \\
\hline Acar clathrata (Defrance, 1816) & $45 c$ & PM, CD \\
\hline
\end{tabular}

Table 1/9. List of the benthic molluscs recorded in Capo Milazzo coastal waters. Sites of collection (1-5) and prevalent habitat of each species are indicated: Photophilic Algae (PA); Phanerogam Meadows (PM); Reef Pools (RP); Coastal Detritic and Biodetritic bottoms (CD); Supratidal Rocky reef (SR). 


\begin{tabular}{|c|c|c|}
\hline species & site & habitat \\
\hline Anadara gibbosa (Reeve, 1844) & 4 & PM \\
\hline Bathyarca pectunculoides (Scacchi, 1835) & $5 \mathrm{~b}$ & $\mathrm{CD}$ \\
\hline Striarca lactea (Linnaeus, 1758) & $15 b$ & $\mathrm{PA}, \mathrm{CD}$ \\
\hline Limopsis tenuis Seguenza, 1876 & $2345 \mathrm{c}$ & $\mathrm{CD}$ \\
\hline Glycymeris bimaculata (Poli, 1795) & 234 & PM \\
\hline Glycymeris glycymeris (Linnaeus, 1758) & 234 & PM \\
\hline Mytilus edulis Linnaeus, 1758 & 3 & AP \\
\hline Mytilus galloprovincialis Lamarck, 1819 & 123 & AP \\
\hline Brachidontes pharaonis (P. Fischer, 1870) & 123 & AP \\
\hline Mytilaster solidus Monterosato, 1883 & 12 & AP \\
\hline Crenella arenaria Monterosato, 1875 ex H. Martin, ms. & $5 c$ & $\mathrm{CD}$ \\
\hline Gregariella petagnae (Scacchi, 1832) & $5 c$ & $\mathrm{CD}$ \\
\hline Gregariella semigranata (Reeve, 1858) & $1245 \mathrm{c}, \mathrm{d}$ & $\mathrm{PA}, \mathrm{PM}, \mathrm{CD}$ \\
\hline Musculus costulatus (Risso, 1826) & $12345 b$ & PA, PM, CD \\
\hline Musculus discors (Linnaeus, 1767) & $5 \mathrm{~d}$ & PA \\
\hline Musculus subpictus (Cantraine, 1835) & $45 b$ & PM, CD \\
\hline Rhomboidella prideauxi (Learch, 1815) & $45 c$ & PM, CD \\
\hline Lithophaga lithophaga (Linnaeus, 1758) & $125 \mathrm{~d}$ & PA \\
\hline Modiolus barbatus (Linnaeus, 1758) & 1234 & PA, PM \\
\hline Gibbomodiola adriatica (Lamarck, 1819) & $25 \mathrm{c}$ & PA, CD \\
\hline Dacrydium hyalinum (Monterosato, 1875) & $35 \mathrm{~b}, \mathrm{c}$ & PM, CD \\
\hline Modiolula phaseolina (Philippi, 1844) & $45 c, d$ & PM, PA, CD \\
\hline Pinna nobilis Linnaeus, 1758 & $2345 \mathrm{~d}, \mathrm{e}$ & PA, PM \\
\hline Pinna rudis Linnaeus, 1758 & 3 & PM \\
\hline Pinctada imbricata radiata (Leack, 1814) & 3 & PM \\
\hline Similipecten similis (Laskey, 1811) & 4 & PM \\
\hline Pecten jacobaeus (Linnaeus, 1758) & $245 \mathrm{c}$ & PM, CD \\
\hline Flexopecten flexuosus (Poli, 1795) & $245 b, c, d$ & PA, PM, CD \\
\hline Flexopecten glaber (Linnaeus, 1758) & $245 \mathrm{~d}$ & PA, PM \\
\hline Flexopecten hyalinus (Poli, 1795) & $245 b$ & PA, PM, CD \\
\hline Aequipecten commutatus (Monterosato, 1875) & $45 \mathrm{~b}$ & PM, CD \\
\hline Aequipecten opercularis (Linnaeus, 1758) & 4 & PM \\
\hline Mimachlamys varia (Linnaeus, 1758) & $245 b$ & $\mathrm{PA}, \mathrm{PM}, \mathrm{CD}$ \\
\hline Palliolum incomparabile (Risso, 1826) & $45 \mathrm{~b} 5 \mathrm{e}$ & PM, CD \\
\hline Pseudamussium clavatum (Poli, 1795) & $45 \mathrm{~b}$ & PM, CD \\
\hline Delectopecten vitreus (Gmelin, 1791) & $5 c$ & $\mathrm{CD}$ \\
\hline Manupecten pesfelis (Linnaeus, 1758) & 4 & PM \\
\hline Talochlamys multistriata (Poli, 1795) & $245 \mathrm{e}$ & PA, PM \\
\hline Spondylus gaederopus Linnaeus, 1758 & $12345 \mathrm{~d}$ & PA, PM \\
\hline Anomia ephippium Linnaeus, 1758 & $12345 \mathrm{~d}, \mathrm{e}$ & PA, PM \\
\hline Heteranomia squamula (Linnaeus, 1758 ) & $5 \mathrm{e}$ & PM \\
\hline Pododesmus patelliformis (Linnaeus, 1761) & $35 \mathrm{~d}$ & PA, PM \\
\hline Lima lima (Linnaeus, 1758) & 23 & PA, PM \\
\hline Limaria hians (Gmelin, 1791) & $245 \mathrm{~d}, \mathrm{e}$ & PA, PM \\
\hline Limaria tuberculata (Olivi, 1792) & $5 b$ & $\mathrm{CD}$ \\
\hline Limatula gwyni (Sykes, 1903) & $45 b$ & PM, CD \\
\hline
\end{tabular}

Table 1/10. List of the benthic molluscs recorded in Capo Milazzo coastal waters. Sites of collection (1-5) and prevalent habitat of each species are indicated: Photophilic Algae (PA); Phanerogam Meadows (PM); Reef Pools (RP); Coastal Detritic and Biodetritic bottoms (CD); Supratidal Rocky reef (SR). 


\begin{tabular}{|c|c|c|}
\hline species & site & habitat \\
\hline Limatula subauriculata (Montagu, 1808) & $5 b$ & $\mathrm{CD}$ \\
\hline Ostrea edulis Linnaeus, 1758 & $1235 \mathrm{c}, \mathrm{d}$ & PA, PM, CD \\
\hline Crassostrea gigas (Thunberg, 1793) & 124 & PA, PM \\
\hline Neopycnodonte cochlear (Poli, 1795) & $45 b, c$ & $\mathrm{PM}, \mathrm{CD}$ \\
\hline Ctena decussata (O.G. Costa, 1829) & $12345 \mathrm{~d}$ & PA, PM \\
\hline Loripinus fragilis (Philippi, 1836) & 234 & PM \\
\hline Loripes orbiculatus Poli, 1791 & 23 & PM \\
\hline Myrtea spinifera (Montagu, 1803) & $45 b$ & PM, CD \\
\hline Lucinoma borealis (Linnaeus, 1767) & 4 & PM \\
\hline Lucinella divaricata (Linnaeus, 1758) & $1345 \mathrm{~d}$ & $\mathrm{PM}, \mathrm{CD}$ \\
\hline Axinulus alleni Carrozza, 1981 & 1 & PM \\
\hline Thyasira biplicata (Philippi, 1836) & 1 & PM \\
\hline Diplodonta intermedia Biondi-Giunti, 1859 & 1 & PM \\
\hline Diplodonta rotundata (Montagu, 1803) & 4 & PM \\
\hline Diplodonta trigona (Scacchi, 1835) & $5 \mathrm{~b}$ & $\mathrm{CD}$ \\
\hline Chama gryphoides Linnaeus, 1758 & 1234 & PA, PM \\
\hline Pseudochama gryphina (Lamarck, 1819) & 234 & PA, PM \\
\hline Kellia suborbicularis (Montagu, 1803) & $5 c$ & $\mathrm{CD}$ \\
\hline Hemilepton nitidum (W.Turton, 1822) & $25 c$ & $\mathrm{PA}, \mathrm{CD}$ \\
\hline Kurtiella bidentata (Montagu, 1803) & $5 \mathrm{~d}$ & PA \\
\hline Epilepton clarkiae (W. Clark, 1852) & 1 & $\mathrm{PA}$ \\
\hline Neolepton sulcatulum (Jeffreys, 1859) & $25 b 5 c$ & $\mathrm{PA}, \mathrm{CD}$ \\
\hline Cardita calyculata (Linnaeus, 1758) & 3 & PM \\
\hline Centrocardita aculeata (Poli, 1795) & $345 b 5 c$ & $\mathrm{PM}, \mathrm{CD}$ \\
\hline Glans trapezia (Linnaeus, 1767) & 234 & PA, PM \\
\hline Coripia corbis (Philippi, 1836) & $5 c$ & $\mathrm{CD}$ \\
\hline Cardites antiquatus (Linnaeus, 1758) & 234 & PA, PM \\
\hline Astarte fusca (Poli, 1791) & $45 c$ & PM, CD \\
\hline Astarte sulcata (da Costa, 1778) & $45 \mathrm{c}$ & PM, CD \\
\hline Digitaria digitaria (Linnaeus, 1758) & $45 d$ & PA, PM \\
\hline Goodallia micalii Giribet \& Peñas, 1999 & $5 \mathrm{c}$ & $\mathrm{CD}$ \\
\hline Goodallia pusilla (Forbes, 1844) & $5 \mathrm{c} 5 \mathrm{~d}$ & $\mathrm{PA}, \mathrm{CD}$ \\
\hline Goodallia triangularis (Montagu, 1803) & $45 \mathrm{c} 5 \mathrm{~d}$ & PM, CD \\
\hline Gonilia calliglypta (Dall, 1903) & $5 \mathrm{c} 5 \mathrm{~d}$ & $\mathrm{CD}$ \\
\hline Acanthocardia paucicostata (G.B. Sowerby II, 1834) & 123 & PM \\
\hline Acanthocardia tubercolata (Linnaeus, 1758) & 3 & PM \\
\hline Parvicardium exiguum (Gmelin, 1791) & 24 & $\mathrm{PA}, \mathrm{PM}$ \\
\hline Parvicardium minimum (Philippi, 1836) & $12345 \mathrm{~d}$ & PM \\
\hline Papillicardium papillosum (Poli, 1791) & $1245 b$ & PM \\
\hline Laevicardium crassum (Gmelin, 1791) & 4 & PM \\
\hline Laevicardium oblongum (Gmelin, 1791) & $45 c$ & PM, CD \\
\hline Cerastoderma edule (Linnaeus, 1758) & 23 & PM \\
\hline Mactra stultorum (Linnaeus, 1758) & 2 & PM \\
\hline Spisula subtruncata (da Costa, 1778) & 14 & PM \\
\hline Moerella distorta (Poli, 1791) & 124 & PM \\
\hline Bosemprella incarnata (Linnaeus, 1758) & 2 & PM \\
\hline
\end{tabular}

Table 1/11. List of the benthic molluscs recorded in Capo Milazzo coastal waters. Sites of collection (1-5) and prevalent habitat of each species are indicated: Photophilic Algae (PA); Phanerogam Meadows (PM); Reef Pools (RP); Coastal Detritic and Biodetritic bottoms (CD); Supratidal Rocky reef (SR). 


\begin{tabular}{|c|c|c|}
\hline species & site & habitat \\
\hline Peronidia albicans (Gmelin, 1791) & 2 & PM \\
\hline Peronaea planata (Linnaeus, 1758) & 2 & PM \\
\hline Moerella pulchella (Lamarck, 1818) & 2 & PM \\
\hline Serratina serrata (Brocchi, 1814) & 24 & $\mathrm{PM}, \mathrm{CD}$ \\
\hline Moerella donacina (Linnaeus, 1758) & $245 d$ & PM \\
\hline Asbjornsenia pygmaea (Lovén, 1846) & 4 & $\mathrm{PM}$ \\
\hline Arcopella balaustina (Linnaeus, 1758) & $45 \mathrm{e}$ & PM \\
\hline Gastrana fragilis (Linnaeus, 1758) & 2 & PM \\
\hline Donax semistriatus Poli, 1759 & $25 \mathrm{~d}$ & PM \\
\hline Donax variegatus (Gmelin, 1791) & $245 \mathrm{~d}$ & PM \\
\hline Gari costulata (W.Turton, 1822) & 4 & $\mathrm{PM}$ \\
\hline Gari depressa (Pennant, 1777) & 3 & PM \\
\hline Gari fervensis (Gmelin, 1791) & 14 & PM \\
\hline Gari tellinella (Lamarck, 1818) & $5 \mathrm{~b}$ & $\mathrm{CD}$ \\
\hline Abra alba (W. Wood, 1802) & $145 \mathrm{~d}$ & PM \\
\hline Abra longicallus (Scacchi, 1835) & 24 & PM \\
\hline Abra nitida (O.F. Müller, 1776) & 12 & PM \\
\hline Abra prismatica (Montagu, 1808) & 14 & PM \\
\hline Abra tenuis (Montagu, 1803 ) & 2 & PM \\
\hline Scrobicularia cottardii (Payraudeau, 1826) & 13 & PM \\
\hline Scrobicularia plana (da Costa, 1778$)$ & 2 & PM \\
\hline Azorinus chamasolen (da Costa, 1778) & 2 & PM \\
\hline Solecurtus scopula (W. Turton, 1822) & 4 & PM \\
\hline Solecurtus strigilatus (Linnaeus, 1758) & 3 & PM \\
\hline Venus casina Linnaeus, 1758 & $45 b$ & PM, CD \\
\hline Venus verrucosa Linnaeus, 1758 & 234 & PM \\
\hline Gouldia minima (Montagu, 1803) & $1345 b$ & $\mathrm{PM}, \mathrm{CD}$ \\
\hline Chamelea gallina (Linnaeus, 1758) & 23 & PM \\
\hline Clausinella fasciata (da Costa, 1778) & 4 & PM \\
\hline Timoclea ovata (Pennat, 1777) & $12345 b$ & PM, CD \\
\hline Pitar rudis (Poli, 1795) & $245 \mathrm{e}$ & PM \\
\hline Callista chione (Linnaeus, 1758) & 1234 & PM \\
\hline Polititapes aureus (Gmeli, 1791) & 2 & PM \\
\hline Polititapes rhomboides (Pennat, 1777) & 2 & PM \\
\hline Ruditapes decussatus (Linnaeus, 1758) & 2 & PM \\
\hline Irus irus (Linnaeus, 1758) & $245 \mathrm{~d}$ & PA, PM \\
\hline Dosinia lupinus (Linnaeus, 1758) & 13 & PM \\
\hline Lajonkairia lajonkairii (Payraudeau, 1826) & $245 a, d$ & PA, PM, RP \\
\hline Lajonkairia substriata Montagu, 1808 & $5 \mathrm{c}$ & $\mathrm{CD}$ \\
\hline Corbula gibba (Olivi, 1792) & $1245 \mathrm{~d}$ & PM \\
\hline Rocellaria dubia (Pennat, 1777) & $1245 b, d$ & PA, PM, CD \\
\hline Hiatella arctica (Linnaeus, 1767) & $25 \mathrm{c}$ & $\mathrm{PA}, \mathrm{CD}$ \\
\hline Hiatella rugosa (Linnaeus, 1767) & $12345 \mathrm{c}$ & PA, PM, CD \\
\hline Xylophaga dorsalis (W. Turton, 1819) & $25 \mathrm{c}$ & PA, CD \\
\hline Bryopa aperta (G.B. Sowerby I, 1823) & $5 \mathrm{~b}$ & $\mathrm{CD}$ \\
\hline Bryopa melitensis (Broderip, 1834) & $5 b$ & $\mathrm{CD}$ \\
\hline
\end{tabular}

Table 1/12. List of the benthic molluscs recorded in Capo Milazzo coastal waters. Sites of collection (1-5) and prevalent habitat of each species are indicated: Photophilic Algae (PA); Phanerogam Meadows (PM); Reef Pools (RP); Coastal Detritic and Biodetritic bottoms (CD); Supratidal Rocky reef (SR). 


\begin{tabular}{|l|l|c|}
\hline \multicolumn{1}{|c|}{ Species } & \multicolumn{1}{c|}{ site } & habitat \\
\hline Cuspidaria cuspidata (Olivi, 1792) & 4 & PM \\
\hline Cardiomya costellata (Deshayes, 1835) & $145 \mathrm{~b}$ & PM, CD \\
\hline SCAPHOPODA & & CD \\
\hline Antalis agilis (M. Sars in G.O. Sars, 1872) & $25 \mathrm{~d}$ & PM \\
\hline Antalis inaequicostata (Dautzenberg,1891) & 24 & PM \\
\hline Antalis vulgaris (da Costa, 1778) & $45 \mathrm{~d}$ & PM \\
\hline Fustiaria rubescens (Deshayes, 1825) & 24 & \\
\hline
\end{tabular}

Table 1/13. List of the benthic molluscs recorded in Capo Milazzo coastal waters. Sites of collection (1-5) and prevalent habitat of each species are indicated: Photophilic Algae (PA); Phanerogam Meadows (PM); Reef Pools (RP); Coastal Detritic and Biodetritic bottoms (CD); Supratidal Rocky reef (SR).

\begin{tabular}{|l|c|}
\hline \multicolumn{1}{|c|}{ Species } & Site \\
\hline Janthina globosa Wainson, 1822 & $2,3,6$ \\
\hline Janthina pallida W. Thompson,1840 & $2,3,6$ \\
\hline Atlanta peronii Lesueur, 1817 & $5 \mathrm{c}$ \\
\hline Cavolinia inflexa (Lesueur, 1813) & $5 \mathrm{~b}, \mathrm{c}$ \\
\hline Diacria trispinosa (Lesueur, 1821) & $5 \mathrm{~d}$ \\
\hline Clio pyramidata Linnaeus, 1767 & $2,5 \mathrm{c}$ \\
\hline Creseis clava (Rang, 1828) & $5 \mathrm{~b}, \mathrm{c}$ \\
\hline Styliola subula (Quoy et Gaimard, 1827) & $5 \mathrm{~b}, \mathrm{c}$ \\
\hline Limacina bulimoides (d'Orbigny, 1835) & $5 \mathrm{c}$ \\
\hline Limacina retroversa (J. Fleming, 1823) & $5 \mathrm{c}$ \\
\hline Limacina trochiformis (d'Orbigny, 1835) & $5 \mathrm{c}$ \\
\hline Heliconoides inflatus (d'Orbigny, 1835) & $5 \mathrm{c}$ \\
\hline Cymbulia peronii Blainville, 1818 & $2,3,6$ \\
\hline Peracle reticulata (d'Orbigny, 1835) & $5 \mathrm{c}$ \\
\hline
\end{tabular}

Table 2. List of the pelagic molluscs recorded in Capo Milazzo coastal waters. Sites of collection of each species $(2$, $3,6)$ are indicated.

\section{CONCLUSIONS}

The check-list of 556 benthic taxa here provided, although not exhaustive of the overall biodiversity of the Milazzo Peninsula, may be considered at least representative of the local mollusc fauna and respective habitats, some of which of relevant conservative values, i.e., phanerogam meadows and the more localized vermetid reefs. Most mollusc species under EEC and National protection, and other ones listed as threatened, occur in the coastal seafloors of the Milazzo peninsula, even in areas submitted to relevant anthropogenic pressure.

In the meantime, the settlement of not indigenous species, mainly of tropical origin, together with disease affecting threatened organisms under EEC protection, testified vulnerability of the local ecosystem toward the global change menaces.

The recent establishment of a marine protected area is an important initiative to protect the most sensitive and most valuable natural habitats, and offers new opportunities to improve their knowledge and conservation. Few data are available today on the local biodiversity of these ecosystems, which should be rapidly investigated in order to evaluate the effect of the protection measures as well as any change in valuable habitats not subject to special protection measures. It must be emphasized, in fact, that the local interdiction to human activities cannot protect the marine reserve against the effects of anthropic pressure on the vast territory, which in the Milazzo area are mainly linked to the presence of oil rigs and related oil tanker traffic.

\section{ACKNOWLEDGMENTS}

The authors wish to thank the relatives of the deceased Mr. Lo Presti, which has provided fishing net remains, Mr. Donato Calapà (Milazzo, Italy) and Mr. Antonino Bitto (Milazzo, Italy) for sampling sediments of 25-35 m depth, and Mr. Walter Renda (Amantea, Cosenza, Italy) for the graphic composition.

\section{REFERENCES}

Battaglia P., Andaloro F., Consoli P., Peda C., Raicevich S., Spagnolo M. \& Romeo T., 2017. Baseline data to characterize and manage the small-scale fishery (SSF) of an oncoming Marine Protected Area (Cape 
Milazzo, Italy) in the western Mediterranean Sea. Ocean \& Coastal Management, 148: 231-244.

Bergamasco A., Culotta L., De Stefano C., Orecchio S., Sammartano S. \& Barreca S., 2014. Composition, distribution and sources of polycyclic aromatic hydrocarbons in sediments of the Gulf of Milazzo (Mediterranean Sea, Italy). Polycyclic Aromatic Compounds, 34: 397-424.

Bombace G., 1969. Appunti sulla malacofauna e sui fondali circalitorali della penisola di Milazzo. Supplemento al bollettino d'informazione Sicilcamere, Ed. Pezzino, Palermo, $56 \mathrm{pp}$.

Bombace G., 1970. Notizie sulla malacofauna e sulla ittiofauna del Coralligeno di falesia. Supplemento Al Bollettino D'informazione Sicilcamere. Ed. Pezzino. Palermo, $77 \mathrm{pp}$.

Cabanellas-Reboredo M., Vázquez-Luis M., Mourre B., Álvarez E., Deudero S., Amores Á., Addis P., Ballesteros K., Barrajón A., Coppa S., García-March J.R., Giacobbe S., Giménez Casalduero F., Hadjioannou L., Jiménez-Gutiérrez S.V., Katsanevakis S., Kersting D., Mačić V., Mavrič B., Patti F.P., Planes S., Prado P., Sánchez J., Tena-Medialdea J., de Vaugelas J., Vicente N., Zohra Belkhamssa F., Zupan I., Hendriks I.E., submitted. Tracking the dispersion of a pathogen causing mass mortality in the pen shell Pinna nobilis: a collaborative effort of scientists and citizens. Scientific Reports.

Consoli P., Romeo T., Giongrandi U. \& Andaloro F., 2008. Differences among fisches assemblages associated with a nearshore vermetid reef and two other rocky habitats along the shores of Cape Milazzo (northern Sicily, central Mediterranean sea). Journal of Marine Biological Association UK., 88: 401-410.

D’Alessandro M., Esposito V., Giacobbe S., Renzi M., Mangano M.C., Vivona P., Consoli P., Scotti G., Andaloro F. \& Romeo T., 2016. Ecological assessment of a heavily human-stressed area in the Gulf of Milazzo, Central Mediterranean Sea: an integrated study of biological, physical and chemical indicators. Marine Pollution Bulletin, 106: 260-273. DOI: 10. 1016/j.marpolbul.2016.01.021.

Di Bella G., Pizzullo G., Bua G.D., Potortì A.G., Santini A. \& Giacobbe S., 2018. Mapping toxic mineral contamination: the southern oyster drill, S. haemastoma (L., 1767), as evaluable sentinel species. Environmental Monitoring Assessment, 190: 1-7. DOI: 10. 1007/s10661-017-6380-x

Di Natale A., 1982. Extra-Mediterranean Species of Mollusca along the Southern Italian Coasts. Malacologia, 22: 571-580.

Gaeta S., Scuderi D. \& Cantone G., 2003. Prime osservazioni sulla selezione dell'habitat in due specie di Haliotis (Mollusca: Gastropoda) del Mediterraneo: H. tuberculata Linnaeus, 1758 e H. stomatiaeformis
Reeve, 1846. Biologia Marina Mediterranea, 10: 561-564.

Geiger D.L., 2000. Distribution and Biogeography of the Recent Haliotidae (Gastropoda: Vetigastropoda) World-wide. Bollettino Malacologico, 35: 1-120.

Giusti F. \& Micali P., 2018. New data on the deep-sea mollusc fauna of the Western Mediterranean (between Capraia island and Cape Corso). Bollettino Malacologico, 54: 61-68.

La Mesa G., Salvati E., Agnesi S. \& Tunesi L., 2017. Assessment of coastal fish assemblages before the establishment of a new marine protected area in the central Mediterranean: its role in formulating a zoning proposal. Mediterranean Marine Science, 18: 1121. DOI: http://dx.doi.org/10.12681/mms. 1788

Lodola A., Nicolini L., Savini D., Deidun A. \& Occhipinti-Ambrogi A., 2013. Range expansion and biometric features of Pinctada imbricata radiata (Bivalvia: Pteriidae) around Linosa Island, Central Mediterranean Sea (Italy). Italian Journal of Zoology, 80: 303-312. DOI: 10.1080/11250003.2013. 775363

Romani L., Giusti F. \& Bogi C., 2016. Nuove segnalazioni di Molluschi batiali per l'Arcipelago Toscano ed aree adiacenti. Bollettino Malacologico, 52: 60-69.

Ruggieri G. \& Greco A., 1965. Studi geologici e paleontologici su Capo Milazzo con particolare riguardo al Milazziano. Geologica Romana, 4: 41-88.

Sarà G., Romano C. \& Mazzola A., 2008. A new Lessepsian species in the western Mediterranean (Brachidontes pharaonis Bivalvia: Mytilidae): density, resource allocation and biomass. JMBA2, Marine Biodiversity Records 1: e8

Scotti G., Consoli P., Esposito V., Chemello R., Romeo T. \& Andaloro F., 2017. Marine caves of the southern tyrrhenian sea: a first census of benthic biodiversity. Journal of Marine Science: Research and Development, 7: 238. DOI: 10.4172/2155-9910.1000238Æ

Sitran R., Bergamasco A., Decembrini F. \& Guglielmo L., 2009. Microzooplankton (tintinnid ciliates) diversity: coastal community structure and driving mechanisms in the southern Tyrrhenian Sea (Western Mediterranean). Journal of Plankton Research, 31: 153-170.

Smriglio C., Prkiæ J., Di Giulio A. \& Mariottini E., 2007. Two new mathildids from the Mediterranean Sea (Gastropoda, Heterobranchia, Mathildidae). Basteria, 71: 177-188.

Templado J., Richter A. \& Calvo M., 2016. Reef building Mediterranean vermetid gastropods: disentangling the Dendropoma petraeum species complex. Mediterranean Marine Science, 17: 13-31.

UNEP-MAP-RAC/SPA, 2006. Reference list of Mediterranean marine habitat types for the selection of sites to be included in the National Inventories of natural 
sites of conservation interest. UNEP-MAP-RAC/SPA, $5 \mathrm{pp}$.

Valdes A., Alexander J., Crocetta F., Yokes M.B., Giacobbe S., Poursanidis D., Zenetos A., Cervera J.L., Ca- baller M., Galil B. \& Schembri P.J., 2013. The origin and dispersal pathway of the spotted sea hare Aplysia dactylomela (Mollusca: Opisthobranchia) in the Mediterranean Sea. Aquatic Invasions, 8: 427-436. 